\title{
ARTICLE OPEN \\ Small heat shock protein CRYAB inhibits intestinal mucosal inflammatory responses and protects barrier integrity through suppressing IKK $\beta$ activity
}

Weimin Xu' ${ }^{1}$, Yuegui Guo ${ }^{1}$, Zhenyu Huang ${ }^{1}$, Haoxin Zhao ${ }^{2}$, Mingxia Zhou ${ }^{3}$, Yuji Huang ${ }^{1}$, Dongpeng Wen ${ }^{1}$, Jinglue Song ${ }^{1}$, Zhehui Zhu ${ }^{1}$, Mingming Sun ${ }^{4}$, Chen-Ying Liu ${ }^{1}$, Yingwei Chen ${ }^{3}$, Long Cui ${ }^{1}$, Xiaolei Wang ${ }^{4}$, Zhanju Liu ${ }^{4}$, Yili Yang ${ }^{2}$ and Peng Du (iD)

Alpha B-crystallin (CRYAB) is an important member of the small heat shock protein family, and plays a protective and therapeutic role in neurological inflammation. CRYAB expression was assessed in cultured HT29 and Caco-2 cells and inflamed mucosa of patients with inflammatory bowel disease (IBD) and colitis models in mice. Lentivirus-overexpressing and CRSIPR/Cas9 systems were used in different cells to upregulate and silence CRYAB expression, respectively. Cell permeable recombined fusion protein TAT-CRYAB was injected intraperitoneally into dextran sulfate sodium (DSS)- or 2,4,6-trinitrobenzene sulfonic acid (TNBS)-induced colitis in mice to assess its anti-inflammatory effects. CRYAB was found to be significantly decreased in the inflamed mucosa from IBD patients and DSS-induced colitis in mice, and negatively correlated with the levels of TNF-a and IL-6, respectively. Enforced expression of CRYAB suppressed expression of proinflammatory cytokines (e.g., TNF-a, IL-6, IL-1 $\beta$, and IL-8) via inhibiting the IKK complex formation, whereas lack of CRYAB expression markedly enhanced proinflammatory responses. Consistently, administration of TAT-CRYAB fusion protein significantly alleviated DSS- or TNBS-induced colitis in mice and protected intestinal barrier integrity. CRYAB regulates inflammatory response in intestinal mucosa by inhibiting IKK $\beta$-mediated signaling and may serve as a novel therapeutic approach in the treatment of IBD.

Mucosal Immunology (2019) 12:1291-1303; https://doi.org/10.1038/s41385-019-0198-5

\section{INTRODUCTION}

Inflammatory bowel disease (IBD) refers to the chronic inflammatory disease that affects the gastrointestinal tract, the most common forms were the ulcerative colitis (UC) and Crohn's disease $(C D){ }^{1}$ Noteworthily, the incidence rate of IBD has been increasing, in particular in the developing countries, ${ }^{2}$ and the disease usually lasts for decades, characterized by alternating periods of the disease activity and remission, ${ }^{3,4}$ thus emphasizing the urgent need for novel and more effective therapeutics. Extensive studies have indicated that the disease arises as a result of the complicated interactions between the genetic and environmental factors, leading to dysregulated immunological responses and tissue damages in the intestine. ${ }^{5-7}$ As such, the etiology and pathology of IBD depend upon the sensitivity of the intestine to inflammatory and immunological stimuli, particularly, the diets and food supplements ingested, and the intestinal microflora and their products. ${ }^{8}$

The rising incidence rate and long-lasting disease course have made IBD a significant challenge for patients and physicians. Present therapeutics, including 5-aminosalicylic acids, glucocorticoids, and immunosuppressive and biological agents, are unsatisfactory or intolerable in a significant percentage of patients. It has been estimated that up to $25-30 \%$ of IBD patients eventually need to undergo surgery due to the ineffectiveness of drugs and disease complications. ${ }^{9}$ Therefore, it is warranted to search for novel candidate target genes for effective clinical treatment.

Alpha B-crystallin, also known as CRYAB or HSPB5, is initially identified as a structural protein of the vertebrate eye lens, and is expressed in a variety of tissues, including the heart, skeletal muscles, brain, and fat. ${ }^{10-12}$ It is a member of small heat shock protein family and functions as a molecular chaperon in order to prevent the aggregation of proteins denatured by stresses, such as radiation and heat shock. ${ }^{10}$ Recent studies have reported that increased expression of $C R Y A B$ renders several highly invasive and metastatic solid tumor cells, ${ }^{13-16}$ whereas degradation and phosphorylation of CRYAB during enterovirus infection facilitates viral replication and induces viral pathogenesis. ${ }^{17}$ Interestingly, several studies have reported that CRAYB plays protective and therapeutic roles in the development of neuroinflammation induced by injury, infection, neurodegeneration, and autoimmunity. $^{18-22}$

In this study, we determined the role of CRYAB in the pathogenesis and treatment of IBD. Taking advantage of intestinal tissues from IBD patients and dextran sulfate sodium (DSS)- and 2,4,6-trinitrobenzene sulfonic acid (TNBS)-induced murine colitis

\footnotetext{
${ }^{1}$ Department of Colorectal Surgery, Xinhua Hospital, Shanghai Jiaotong University School of Medicine, Shanghai 200092, China; ${ }^{2}$ Suzhou Institute of Systems Medicine, Center for

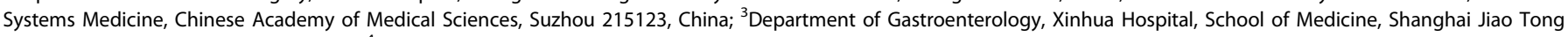
University, Shanghai 200092, China and ${ }^{4}$ Department of Gastroenterology, The Shanghai Tenth People's Hospital, Tongji University, Shanghai 200072, China Correspondence: Zhanju Liu (liuzhanju88@126.com) or Yili Yang (yyl@ism.cams.cn) or Peng Du (dupeng@xinhuamed.com.cn)

These authors contributed equally: Weimin Xu, Yuegui Guo, Zhenyu Huang
}

Received: 2 January 2019 Revised: 3 August 2019 Accepted: 12 August 2019

Published online: 3 September 2019 
models, we demonstrated that expression of CRYAB was decreased in the inflamed mucosa of IBD patients and negatively correlated with the inflammatory reaction. We also found that CRAYB inhibited inflammation by hampering IKK $\beta$-mediated signaling, and that administration of TAT-CRYAB recombined protein alleviated DSS-induced intestinal mucosal inflammation in mice. These results indicate that CRYAB protects intestinal mucosa from inflammation and provides the first evidence that exogenous CRYAB may act as a potential therapeutic approach for the treatment of IBD.

\section{MATERIALS AND METHODS}

Patients and specimens

Non-inflamed and inflamed colon mucosa were collected from 30 patients with active UC (A-UC) and 33 patients with active $C D$ (A-CD) who underwent surgery or colonic biopsy at the Department of Colorectal Surgery, Xinhua Hospital, Shanghai Jiaotong University School of Medicine (Shanghai, China) from February 2008 to March 2017. Moreover, normal colon tissues were obtained from 45 patients who underwent colonic biopsy for colon polyps as controls (HC). In addition, paired inflamed and distant non-inflamed colon tissues were collected from six patients with active UC, and six patients with active CD. The diagnosis of CD and UC was based on clinical, endoscopic, radiological examination, and histological changes. The study was approved by the Ethics Committee of Xinhua Hospital (No. XHEC-D-2018-054). All patients signed an informed consent prior to the study.

Cell culture and treatment

The human monocyte cell line THP-1, colonic adenoma cell lines HT29 and Caco-2, colorectal adenocarcinoma cell lines HCT116 and SW480, and HEK293 cell line were obtained from the American Type Culture Collection (Manassas, VA, USA). These cell lines were cultured with the Dulbecco's modified Eagle's medium (DMEM) supplemented with $10 \%$ fetal bovine serum (Gibco, Shanghai, China), $100 \mu \mathrm{g} / \mathrm{ml}$ of penicillin, and $100 \mathrm{U} / \mathrm{ml}$ of streptomycin at $37^{\circ} \mathrm{C}$ with $5 \% \mathrm{CO}_{2}$.

Enforced expression of CRYAB in intestinal epithelial cells The CDNA of CRYAB was cloned into puromycin-resistant lentiviral vector (pLVX-Puro). The lentivirus particles were prepared using HEK293T cells according to the manufacturer's protocol (Takara, Beijing, China). THP-1, HT29, and Caco-2 cells were then seeded into six-well culture plates and incubated overnight, infected with CRYAB-expressing lentivirus, and were then treated with puromycin to select the cells overexpressing CRYAB in a stable manner.

Silencing CRYAB expression using CRISPR/Cas9 system

The guide RNA targeting CRYAB and Cas9 was expressed in HT29 cells using the Lenti-CRISPR V2 vector. CRYAB-deficient cells were then selected with puromycin, examined using qRT-PCR and immunoblotting, and verified by genomic sequencing.

\section{Immunohistochemistry and immunofluorescence}

Paraffin-embedded tissues were deparaffinized in xylene, rehydrated with descending concentrations of ethanol, and the antigen was retrieved by heating in a citrate buffer ( $\mathrm{pH}$ 6.0). After treating with $3 \%$ hydrogen peroxide and $5 \%$ goat serum, the slides were incubated with specific antibodies at $4{ }^{\circ} \mathrm{C}$ overnight. After thorough washing with PBS, the slides were incubated with HRP-labeled secondary antibody for $30 \mathrm{~min}$ at room temperature and visualized with diaminobenzidine chromogen. For immunofluorescence staining, AlexaFluor 594-conjugated and $\mathrm{SABC}$-cy3-conjugated anti-rabbit lgGs were used as the secondary antibodies.
Immunofluorescence staining of cultured cells

Cells grown on the cover slides were fixed with cold $4 \%$ paraformaldehyde for $30 \mathrm{~min}$, and were permeabilized by exposing to $0.3 \%$ Triton X-100 in cold PBS for 30 min. After blocking in $5 \%$ bovine serum albumin (BSA) for $30 \mathrm{~min}$ at room temperature, the cells were incubated with anti-NF-KB p65 mAb (1:100, diluted in $3 \% \mathrm{BSA}$ ) for $2 \mathrm{~h}$ at room temperature. After thorough washing with PBS containing $0.1 \%$ Triton X-100, these cells were incubated with AlexaFluor 594-conjugated donkey anti-rabbit IgG (Life Technologies, Grand Island, NY, USA) in the dark for $1 \mathrm{~h}$ at room temperature, then counter-stained with $5 \mu \mathrm{g} / \mathrm{ml} \mathrm{DAPI}$, and were eventually analyzed using a fluorescence microscopy.

\section{Quantitative real-time PCR (qRT-PCR)}

The total RNA was extracted from the cultured cells and primary tissues using TRIzol reagent (Invitrogen, Carlsbad, CA, USA) according to the manufacturer's instructions. Reverse transcription was performed from $1 \mu \mathrm{g}$ of the total RNA using PrimeScript ${ }^{{ }^{M}}{ }_{\text {RT }}$ Master Mix (Takara). qRT-PCR was carried out using the SYBR Premix ExTaq ${ }^{\mathrm{TM}}$ (Takara) and an Applied Biosystems 7500 Fast real-time PCR system (公司). Relative expression of mRNA was evaluated by $2^{-\Delta \Delta \mathrm{Ct}}$ method, and was normalized to the expression of GAPDH. All experiments were repeated in triplicates. Sequences of PCR primers in this study are listed in Supplementary Table 1.

\section{Immunoblotting}

Cells were lysed with 1\% NP40 lysis buffer supplemented with $\mathrm{NaF}, \mathrm{Na}_{3} \mathrm{VO}_{4}$, and protease inhibitor cocktail. Lysates comprising equal amounts of protein $(20 \mu \mathrm{g} /$ well $)$ were loaded and separated by SDS-PAGE, and were then transferred onto the nitrocellulose membrane. The membrane was blocked with 5\% BSA for at least $1 \mathrm{~h}$, and was then incubated with a specific antibody at $4{ }^{\circ} \mathrm{C}$ overnight. After washing the membranes three times with trisborate saline containing $0.1 \%$ tween- 20 for $5 \mathrm{~min}$ each, the specific protein was visualized with HRP-conjugated secondary antibody and enhanced chemiluminescence.

\section{Co-immunoprecipitation assay (Co-IP)}

HEK293T cells were transfected with the plasmids expressing IKBaFlag, Flag-IKK $\beta$, and CRYAB-HA or the control plasmid pCDNA3.1 as indicated. After $24 \mathrm{~h}$ of culture, the cells were lysed with $0.3 \%$ NP40 lysis buffer supplemented with $\mathrm{NaF}, \mathrm{Na}_{3} \mathrm{VO}_{4}, \mathrm{PMSF}$, and protease inhibitor cocktail at $4{ }^{\circ} \mathrm{C}$ for $30 \mathrm{~min}$. The lysates were prepared by centrifugation at $12,000 \mathrm{rpm}$ for $15 \mathrm{~min}$ at $4{ }^{\circ} \mathrm{C}$, and were then subjected to immunoprecipitation by incubating with anti-Flag or anti-HA antibodies conjugated with agarose for $3 \mathrm{~h}$. Following extensive washes with TBST, the agarose beads were then boiled with $2 \times$ loading buffer for $5 \mathrm{~min}$. The supernatants were separated on SDS-PAGE, and were analyzed via immunoblotting by using anti-HA and anti-FLAG antibodies.

\section{ELISA}

Human TNF- $\alpha$, IL-6, IL-1 $\beta$, and IL-8 in the culture supernatants were measured using enzyme-linked immunosorbent assay (ELISA) kits purchased from Invitrogen with the Quick EIATM ELISA protocol, according to the manufacturer's protocol.

\section{Determination of IKK $\beta$ activity}

IKK $\beta$ activity was determined via the phosphorylation of specific peptide (RRREEEEESAAA) in the presence and absence of a specific small molecular inhibitor (5-phenyl-3-ureido)-thiophene-2-carboxamide, according to the manufacturer's instructions from Shanghai Haling Biotechnology (Shanghai, China).

Dual-luciferase reporter assays

The plasmids pNFKB-Luc and ( $1 \mu \mathrm{g} / 3$ well) and TK-Renilla ( $20 \mathrm{ng} /$ well) were co-transfected into different cells cultured in a 24-well 
plate with lipofectamine 2000 reagent (Invitrogen). After the indicated treatment, the cells were lysed with the passive lysis buffer, and the luciferases were evaluated using the dualluciferase reporter assay kit (Promega, Madison, WI, USA) according to the manufacturer's instruction.

Mice and DSS-induced murine colitis model

The 6-week-old C57BL/6 male mice $(20 \mathrm{~g})$ were purchased from the Experimental Animal Center of the Chinese Academy of Sciences (Shanghai, China) and were maintained in the animal facility of the Xinhua Hospital, Shanghai Jiaotong University School of Medicine. To induce colitis, 8-week-old male mice were fed with $3.5 \%$ of DSS in drinking water and were monitored for their body weight, activity, and conditions, such as diarrhea and bloody stools. All mice were killed after 7-day induction. All animal experimental procedures were approved by the Laboratory Animal Care and Welfare Committee of Xinhua Hospital (No. XHEC-F-2018-017).

\section{2,4,6-trinitrobenzene sulfonic acid (TNBS)-induced colitis}

The 8-week-old C57BL/6 male mice $(25 \mathrm{~g})$ were used for the induction of TNBS-induced colitis. They were first sensitized with $1 \%$ TNBS solution (presensitization solution) diluted from 5\% TNBS solution with acetone and olive oil mixture (4:1 volume ratio). The presensitization solution $(100 \mu \mathrm{l})$ was applied to a $1 \times 1 \mathrm{~cm}^{2}$ shaved area on the back of each mice. After 7 days, each mouse was anesthetized with $2.5 \%$ chloral hydrate solution and given $150 \mu \mathrm{l} 2.5 \%$ TNBS solution intrarectally by using a catheter through the anus. The activities and defecation of mice were assessed every day for consecutive 3 days, then killed for further examination.

Intestinal integrity examined by fluorescein isothiocyanate (FITC)dextran

FITC-dextran was utilized to examine the intestinal integrity in DSS and TNBS-induced mice colitis models. The mice were given $150 \mu \mathrm{L}(80 \mathrm{mg} / \mathrm{mL})$ of FITC-dextran in sterile water at the last day of induction (the seventh day of DSS induction or the third day of TNBS induction). Blood samples $(100 \mu \mathrm{L})$ were then collected from the tail vein to measure the amounts of FITC fluorescence (480 excitation/520 emission), which reflected the relative epithelial permeability.

\section{RNA and protein extraction from mouse colon}

Mice were euthanized to dissect the colon and rectum. After extensive washing with pre-cooled PBS, the intestinal tissues were cut and homogenized to extract RNA with TRIzol reagent and proteins with RIPA lysis buffer supplemented with protease inhibitors cocktail, respectively, using the methods as described above.

Disease activity index (DAI) and histological pathological scoring To assess DAl score of each mouse, body weight, stool character, and occult or rectal bleeding were monitored daily after DSS or TNBS administration, which were scored on a scale of 0-4. DAI for each mouse was calculated by dividing the total scores by 3 as listed in Supplementary Table 2. ${ }^{23}$ The histopathological scores of mice with induced inflammations were assessed based on neutrophils infiltration, crypts, cross-section involvement, and erosion or ulceration formation by two independent pathologists on the scale of $0-3$. The detailed scores for mice are listed in Supplementary Table $3 .^{24}$

Production of TAT-CRYAB fusion protein

Synthetic DNA coding the TAT-PTD was inserted into the Nhe I site of a pET28a vector. The CRYAB CDNA was then subcloned into the BamH I and EcoR I sites of the pET28a-PTD plasmid. Sequence of the resulted TAT-CRYAB-expressing construct was verified via DNA sequencing. The production and purity of the recombinant protein was examined by SDS-PAGE and Coomassie blue staining.

Statistical analysis

The data were presented as mean \pm standard deviation (SD). Graph Pad Prism 5 software (San Diego, CA, USA) and SPSS Statistics version 19.0 software (IBM 2010, Chicago, IL, USA) were used for statistical analyses. Unpaired Student's $t$ test was used to compare the difference between two single groups. Pearson's correlation test was performed to analyze the relationship between the levels of TNF- $a$ and IL-6 and CRYAB expression in the inflamed mucosa of IBD patients. All statistical tests were twosided and considered as significant with a $p$-value of $<0.05$.

\section{RESULTS}

CRYAB expression is significantly decreased in inflamed colon from IBD patients

To study the molecular mechanisms of CRYAB involved in the development and pathogenesis of IBD, we initially examined CRYAB expression in the inflamed colon from patients with A-UC $(n=30)$ and A-CD $(n=33)$ by qRT-PCR. A significant decrease in the CRYAB mRNA was observed in the inflamed colon from patients with A-UC and A-CD compared with that from the normal colons ( $n=45$; Fig. 1a). Moreover, we also observed that the expression of IL- 6 and TNF- $a$ was notably increased in the inflamed tissues of UC and CD (Fig. 1a), and that their levels were negatively correlated with the CRYAB expression in the inflamed colon from patients with A-CD (for TNF- $a$, Spearman's rank correlation coefficient, $R=-0.4744, p=0.0061$; for IL-6, $R=$ $-0.4631, p=0.0067$; Fig. $1 b)$. Nevertheless, no significant correlation was observed between TNF- $a$ or IL- 6 and CRYAB in the inflamed mucosa from A-UC patients, presumably due to the wide dispersion in the levels of TNF- $\alpha$ and IL- 6 . We further examined the CRYAB expression by immunoblotting in paired, inflamed, and unaffected tissues from six UC and six $C D$ patients. When compared with the paired normal mucosa, CRYAB was markedly decreased in the inflamed mucosa of UC and CD patients (Fig. 1c). Furthermore, a significant increase in phosphorylated p65 and $\mathrm{IKBa}$, indicative of activated proinflammatory NF-KB signaling, was also observed in these tissues (Fig. 1C). This was further confirmed by immunohistochemical analysis of the $C D$ tissues. The levels of CRYAB were found to be notably lower in the inflamed tissues of $\mathrm{CD}$ patients compared with controls, which was also accompanied by an increased infiltration of the macrophages and $T$ lymphocytes in inflamed mucosa (Fig. 1d). In addition, immunofluorescence staining for intestinal epithelial marker CK18, macrophage marker $C D 68$, and $C R Y A B$ was performed and identified that macrophages but not intestinal epithelial cells mainly expressed $C R Y A B$ in inflamed tissues from $C D$ patients (Supplementary Fig. 1). Detailed clinical characteristics of IBD patients are listed in Supplementary Table 4.

CRYAB inhibits production of proinflammatory cytokines by LPSinduced macrophages

To examine whether CRYAB was able to affect the production of proinflammatory cytokines, we cultured THP-1 cells in vitro and transfected with CRYAB-expressing lentivirus (Fig. 2a). These cells were then stimulated with LPS under different conditions in order to assess the production of proinflammatory cytokines (e.g., TNF-a, IL- 6 , IL-1 $\beta$, and IL-8) by qRT-PCR and ELISA. As shown in Fig. $2 b$, the mRNA levels of these cytokines were rapidly induced in THP-1 cells transfected with control lentiviruses, while they were significantly reduced in CRYAB-expressing lentivirus-transfected cells. The same pattern of cytokine expression was found in these cells when analyzed by ELISA (Fig. 2c).

Moreover, we examined the effects of CRYAB on LPSstimulated phosphorylation of IKBa and p65 in THP-1 cells using 

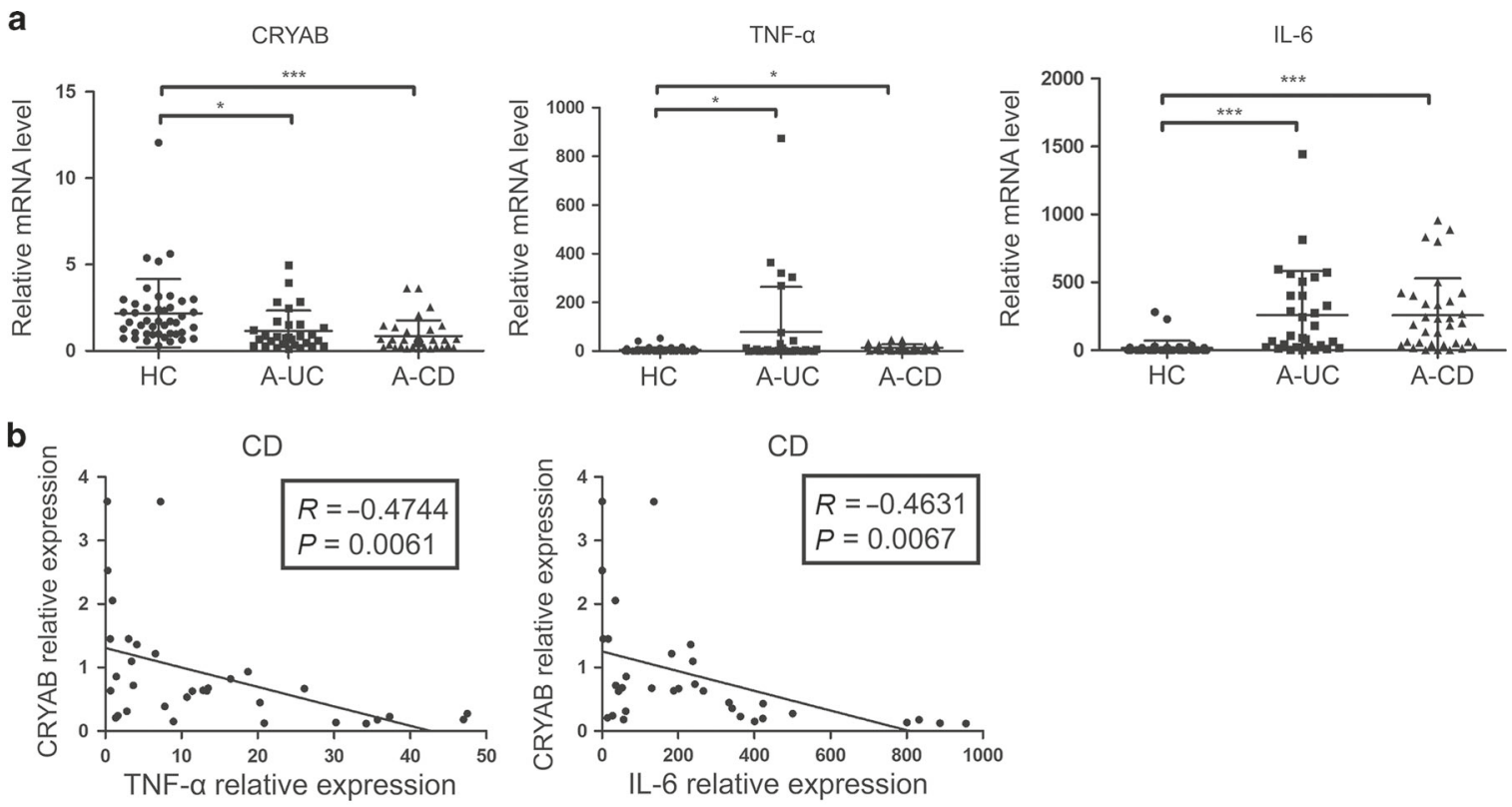

C
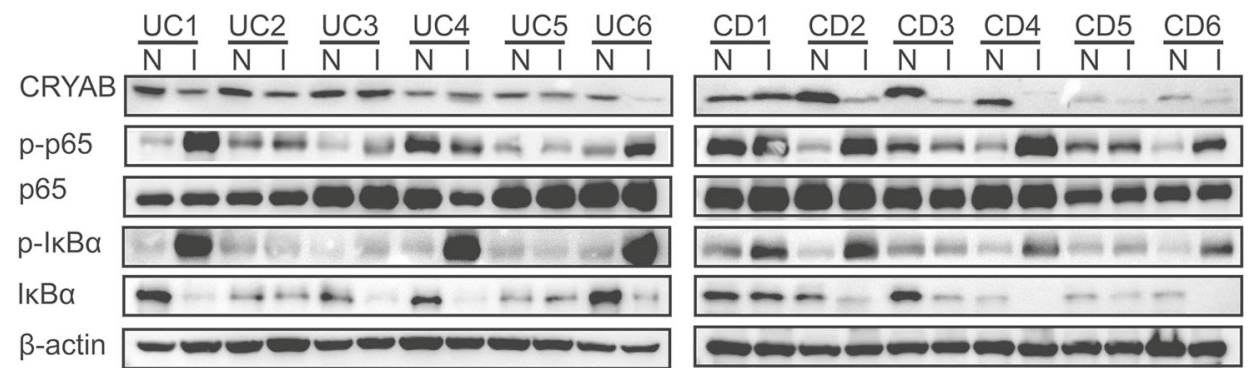

d
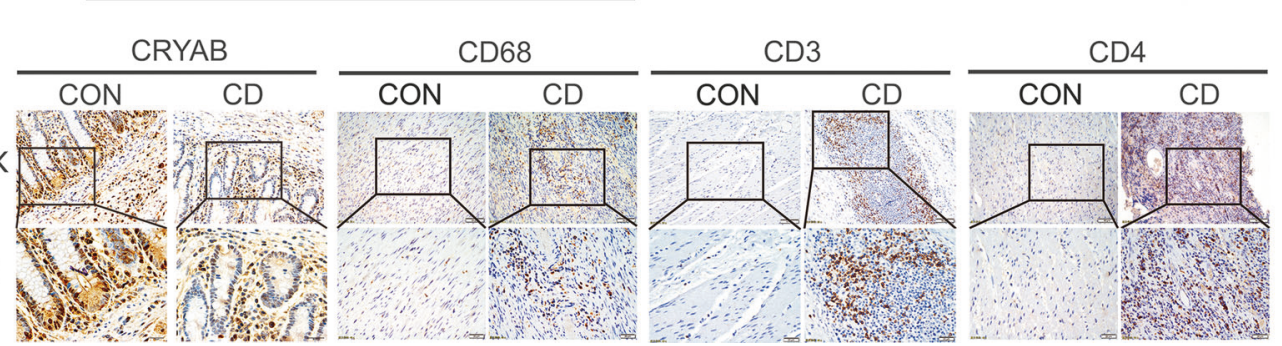

Fig. 1 CRYAB is decreased in the inflamed colon tissues from IBD patients. a qRT-PCR analysis of CRYAB, TNF- $\alpha$, and IL-6 expression. RNA was isolated from healthy controls (HC, $n=45$ ), active UC patients (A-UC, $n=30)$, and active CD patients (A-CD, $n=33$ ). CRYAB was significantly decreased, whereas TNF- $\alpha$ and IL- 6 were markedly increased in A-UC and A-CD than those in HC, $\left({ }^{*} p<0.05,{ }^{* *} p<0.001\right)$. b Relationships between TNF- $\alpha$, IL- 6 , and CRYAB mRNA in inflamed mucosa from 33 A-CD. Significant correlations existed between the levels of TNF- $\alpha$ mRNA and CRYAB (Spearman's rank correlation coefficient, $R=-0.4744, p=0.0061$ ), and IL- 6 and CRYAB expression (Spearman's rank correlation coefficient, $R=-0.4631, p=0.0067)$. c The expression of CRYAB, p65, $\mathrm{p}-\mathrm{p} 65$, I $\mathrm{\kappa} B \alpha$, and $\mathrm{p}-\mathrm{l} \kappa \mathrm{B} \alpha$ in the inflamed and unaffected tissues from six paired A-UC and six paired A-CD patients was examined by immunoblotting with specific antibodies. d Representative immunohistochemical images of CRYAB, CD68, CD3, and CD4 in the paired, inflamed, and unaffected tissues of the same CD patients (magnification: $\times 200$, upper panels; $\times 400$, lower panels). All data are shown from three independent experiments

phosphorylated protein-specific immunoblotting. As presented in Fig. 2d, CRYAB significantly reduced LPS-stimulated phosphorylation of IKBa and $p 65$, in particular, during $1-6 \mathrm{~h}$ after the stimulation. Thus, CRYAB appears to block the activation of NF-KB and suppresses the production of proinflammatory cytokines in macrophages at transcriptional levels.

CRYAB regulates immune response of HT29 and Caco-2 cells in response to TNF-a

To explore whether CRYAB is essential in regulating intestinal cells in response to inflammatory stimulation, we examined CRYAB expression in HT29 and Caco-2 cells at the mRNA and protein levels. Both epithelial cell lines expressed moderate levels of
CRYAB at the baseline, which significantly decreased under stimulation with TNF-a in a time-dependent manner (Fig. 3a), presumably recapitulating reduced $C R Y A B$ in intestinal tissues from patients with UC and CD. Furthermore, we assessed the antiinflammatory effects of enforced expression of CRYAB on HT29 and Caco-2 cells in response to TNF-a (Fig. 3b). As presented in Fig. 3c-f, TNF- $a$ was found to promote the expression of TNF-a, IL-6, IL-1 $\beta$, and IL-8 mRNAs in HT29 and Caco-2 cells. When compared with the cells infected with control lentivirus, CRYABexpressing lentivirus-transfected cells expressed a marked decrease of mRNAs of these cytokines after exposure to TNF-a, notably at $12-24 \mathrm{~h}$. The inhibition of inflammatory cytokines in HT29 and Caco-2 cells by CRYAB overexpression was also 
a

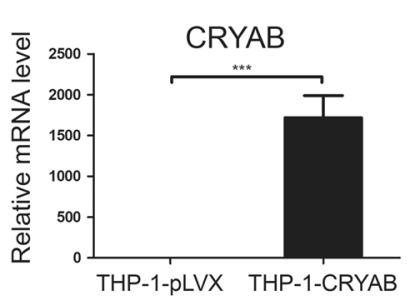

b

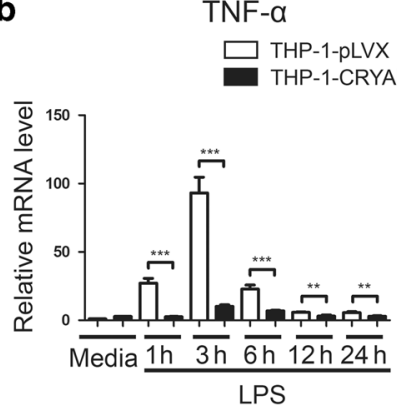

C

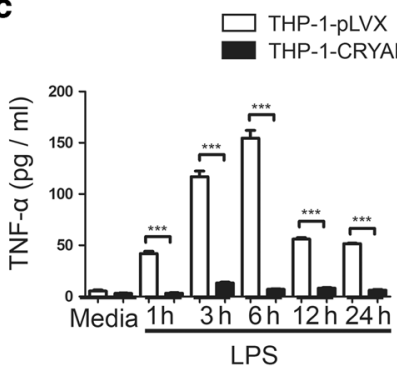

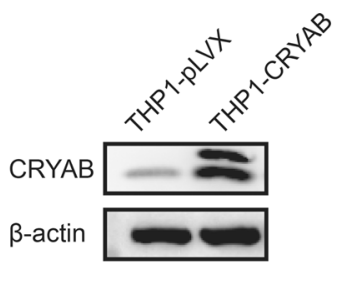

IL-6
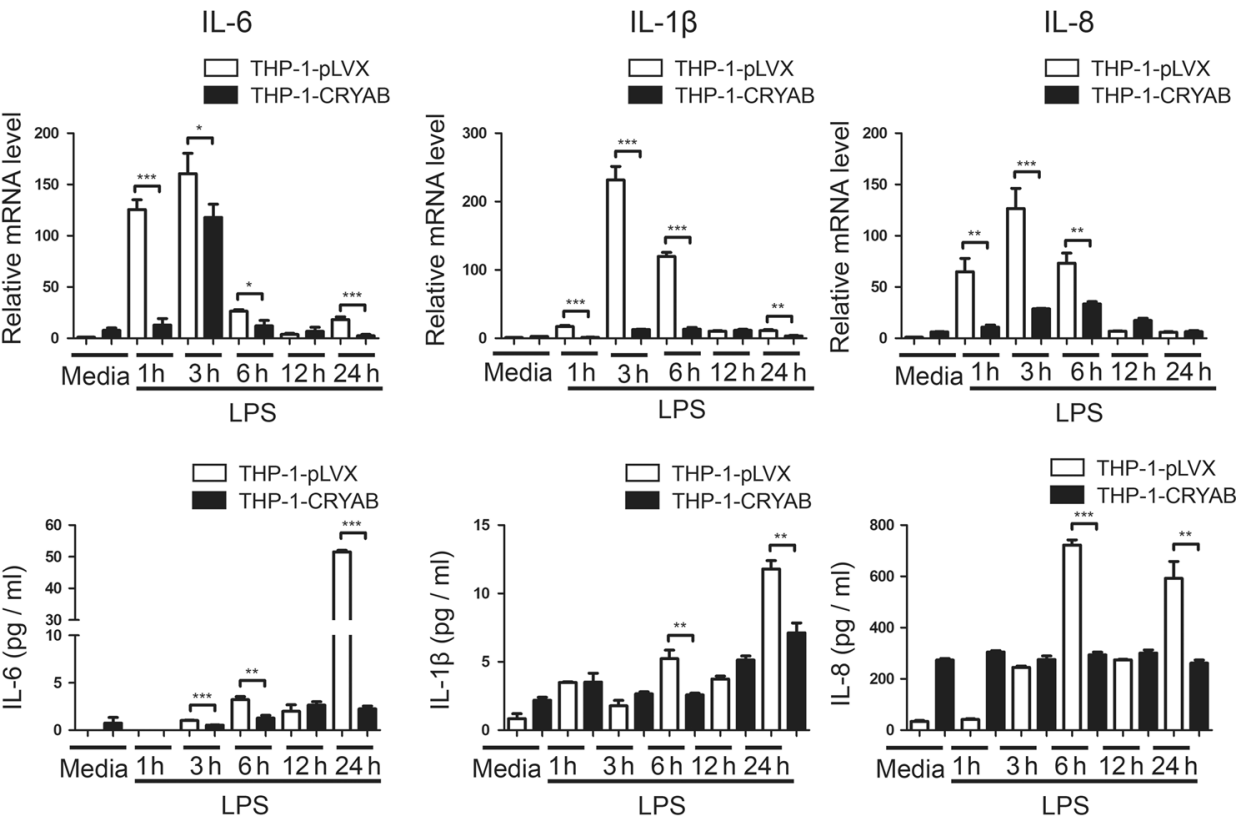

d

THP-1-CRYAB

$\frac{\text { Media }}{-+} \frac{\text { LPS-1h }}{-+} \frac{\text { LPS-3h }}{-+} \frac{\text { LPS-6h }}{-+} \frac{\text { LPS-12h }}{-+} \frac{\text { LPS-24h }}{-+}$

THP-1-pLVX

$p-\mid k B a$

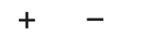

$+-+-+-$

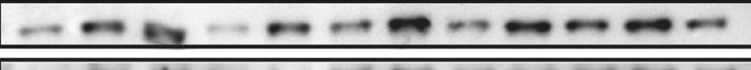

$37 \mathrm{KDa}$

IKBa

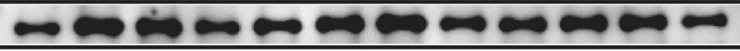

37KDa

p-p65

p65

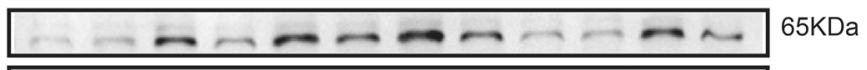

$\beta$-actin

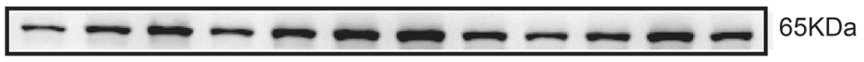

$\beta$-actin

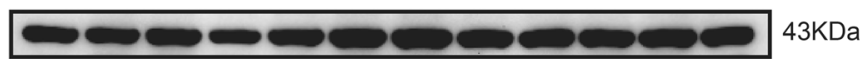

Fig. 2 Enforced expression of CRYAB inhibits LPS-induced production of inflammatory cytokines by THP-1 cells. a Expression of CRYAB in THP1 cells transfected with CRYAB-expressing lentivirus was detected by qRT-PCR and immunoblotting. $\mathbf{b}$, $\mathbf{c}$ Cytokine production in THP-1-pLVX and THP-1-CRYAB cells after LPS $(100 \mathrm{ng} / \mathrm{ml})$ stimulation for indicated time. RNA was isolated to assess the mRNA levels of proinflammatory cytokines (e.g., TNF- $\alpha$, IL-6, IL-1 $\beta$, and IL-8) by qRT-PCR. b Cytokines in the supernatants were measured by EILSA. ${ }^{*} p<0.05,{ }^{* *} p<0.01,{ }^{* * *} p<$

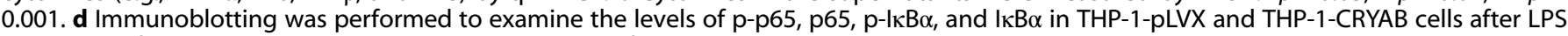
stimulation for the indicated times. All data are shown from three independent experiments

confirmed by ELISA (Fig. 3g). Moreover, we utilized the CRSPR/ Cas9 system to downregulate CRYAB expression in HT29 cells (Supplementary Fig. 2A, B). Compared with controls, TNF-ainduced expression of TNF- $a$, IL-6, and IL-8 mRNAs was significantly increased in CRYAB-deficient cells (Supplementary Fig. 2C). Collectively, these data indicate that CRYAB is an endogenous anti-inflammatory molecule in regulating immune responses of intestinal epithelial cells.

CRYAB interacts with IKK $\beta$ and IKKa and inhibits IKK $\beta$ activity As shown in Fig. 2d, ectopic expression of CRYAB in THP-1 cells inhibited LPS-induced activation of the NF-KB pathway. We also found that TNF-a-induced phosphorylation and subsequent downregulation of IKBa were markedly attenuated by enforced expression of CRYAB in HT29 and Caco-2 cells (Fig. 4a). Consistent with these changes, CRYAB expression decreased TNF-a-induced luciferase expression driven by the NF-KB response element (Fig. 4b), resulting in blocking of translocation of p65 from the cytoplasm to the nucleus (Fig. 4c). Furthermore, the knockout of CRYAB in HT29 and Caco-2 cells significantly increased the levels of p-p65 and p-IKBa following TNF-a stimulation (Supplementary Fig. 2D). These results indicate that $C R Y A B$ inhibits intestinal inflammation by blocking the activation of the NF-KB pathway.

As IKB kinase (IKK) $\beta$ is crucial in phosphorylating IKB to activate $N F K B$, we asked whether CRYAB could affect its action. As shown in Fig. $5 \mathrm{a}$, transfection of IKK $\beta$-expression plasmid into HCT116 and SW480 cells led to $\mathrm{p} 65$ and IKB phosphorylation and IKB degradation, whereas co-expression of CRYAB markedly reduced 
a

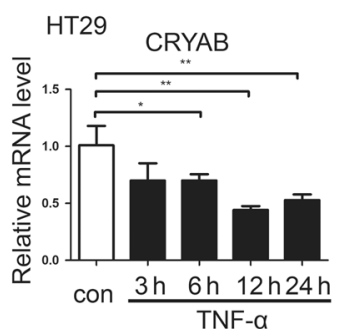

HT29

con $3 \mathrm{~h} 6 \mathrm{~h} 12 \mathrm{~h} 24 \mathrm{~h}$

CRYAB

$\beta$-actin

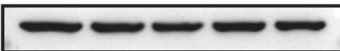

Caco-2 CRYAB

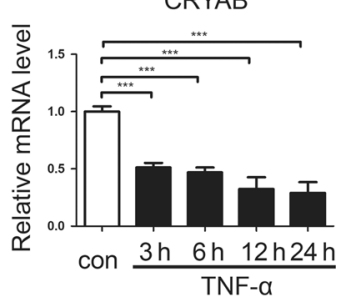

Caco-2 TNF- $\alpha$
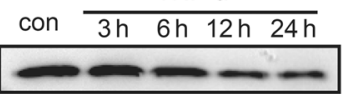

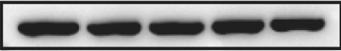

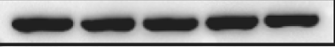

C

TNF- $\alpha$
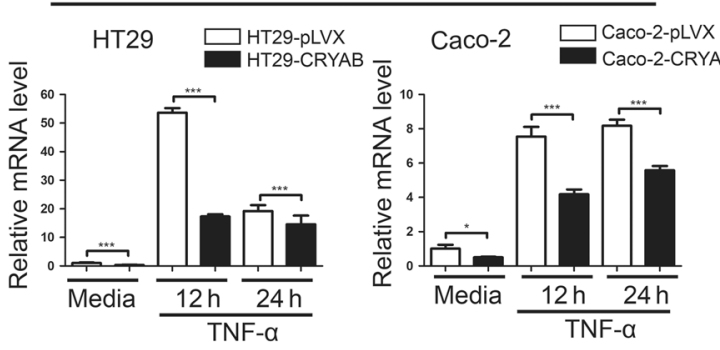

e
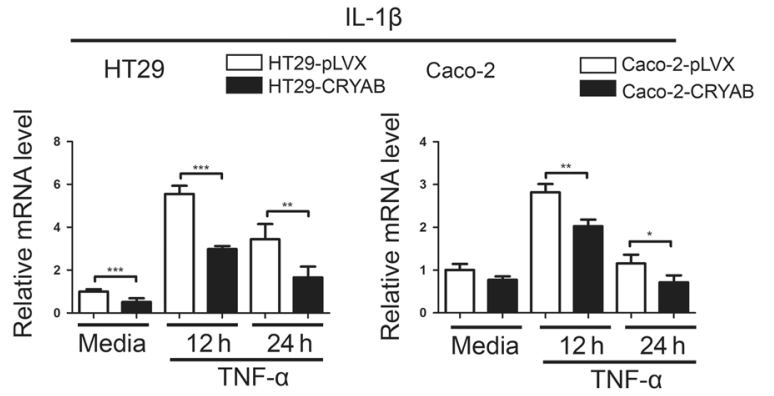

b

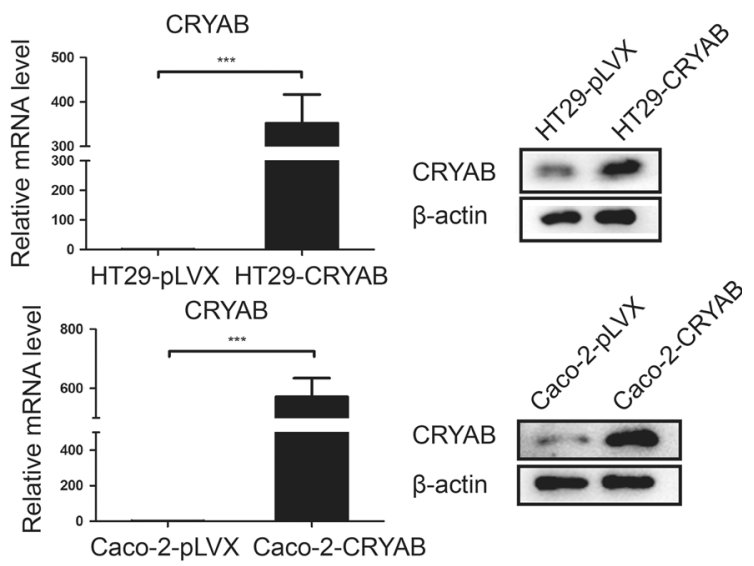

d

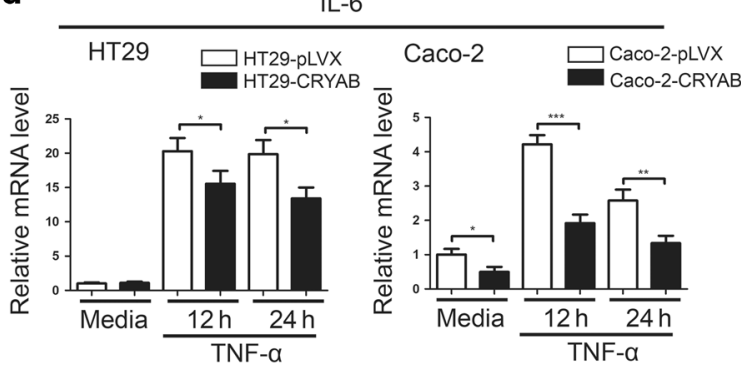

f
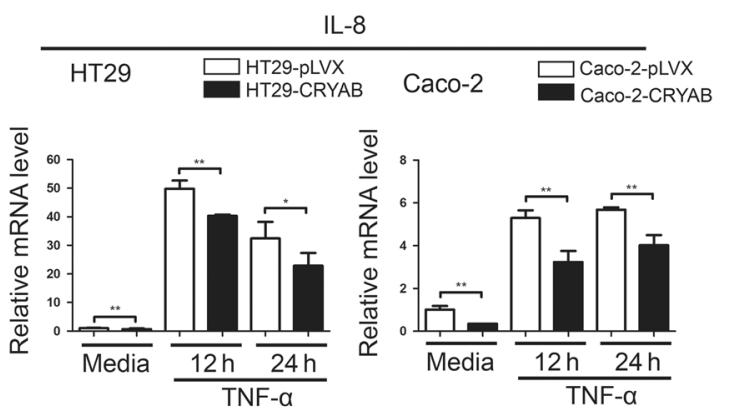
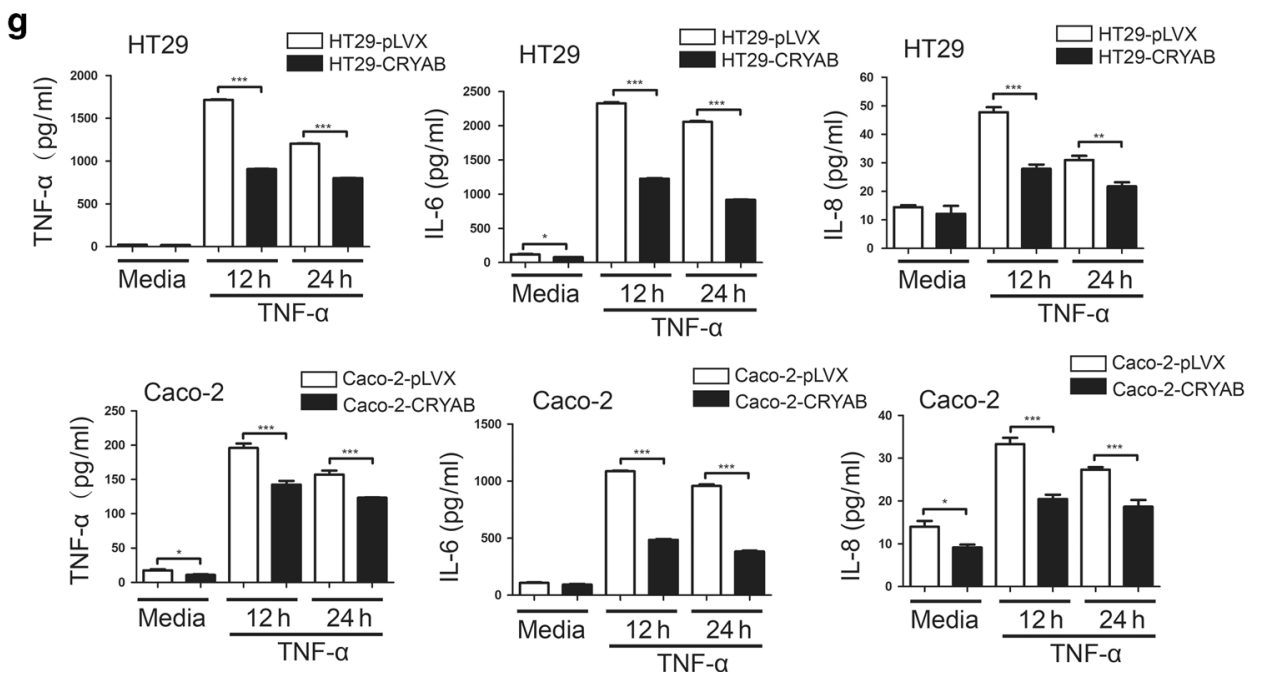

Fig. 3 Enforced expression of CRYAB inhibits TNF- $\alpha$-induced inflammatory response in HT29 and Caco-2 cells. a TNF- $\alpha$ decreased CRYAB in HT29 and Caco- 2 cells. The cells were treated with TNF- $\alpha(10 \mathrm{ng} / \mathrm{ml})$ for indicated times. CRYAB expression was assessed by qRT-PCR and immunoblotting; $p<0.05,{ }^{* *} p<0.01,{ }^{* * *} p<0.001$. b HT29 and Caco-2 cells were transfected by CRYAB-expressing lentivirus. CRYAB level was assessed by qRT-PCR and immunoblotting; ${ }^{* * *} p<0.001$. c-f Expression of TNF- $\alpha, \mathrm{IL}-6, \mathrm{IL}-1 \beta$, and IL-8 was examined in CRYAB-overexpressing and control cells after exposed to TNF- $\alpha(10 \mathrm{ng} / \mathrm{ml})$ for indicated times; ${ }^{*} p<0.05,{ }^{* *} p<0.01,{ }^{* * *} p<0.001$. g Cytokines in the supernatants were measured by EILSA. ${ }^{* *} p<0.01,{ }^{* * *} p<0.001$. All data are shown from three independent experiments 
a

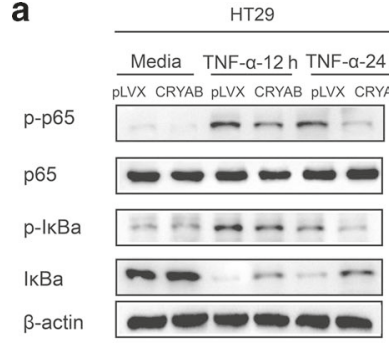

b

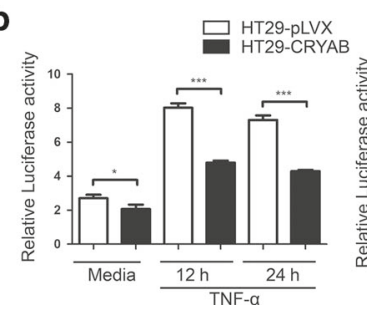

Caco-2
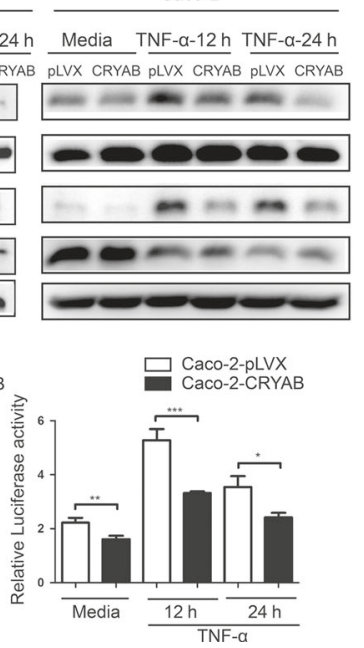

c

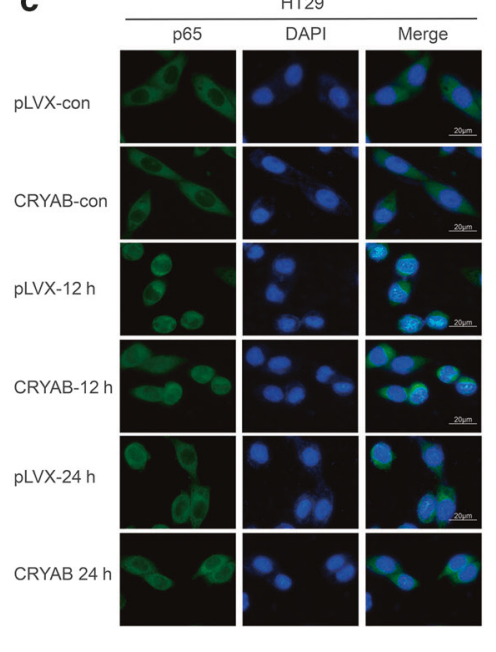

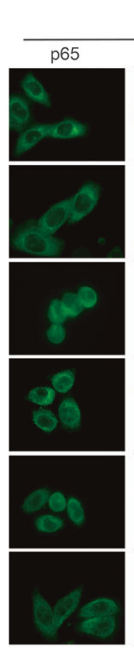

Caco-2

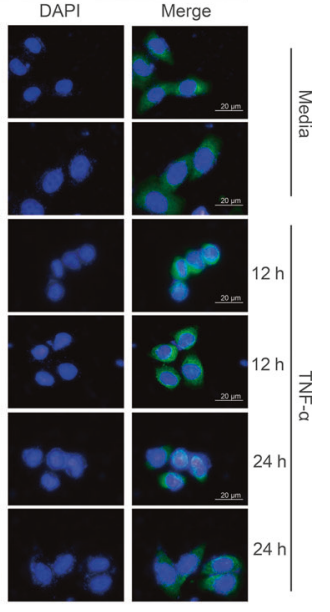

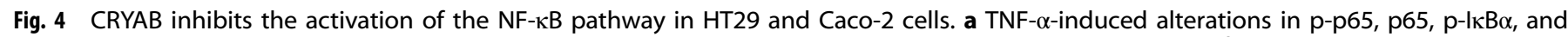
I $\mathrm{B} \alpha$ were examined by immunoblotting in the CRYAB-overexpressing and control cells. $\mathbf{b}$ NF- $\mathrm{NB}$-driven luciferase was inhibited by CRYAB. CRYAB-overexpressing and control cells were transfected with a NF- $\mathrm{kB}$-driven luciferase reporter. Dual-luciferase assays were performed after TNF- $\alpha$ stimulation $(10 \mathrm{ng} / \mathrm{ml})$ for indicated times. The firefly luciferase activity was measured with relative normalization for Renilla; ${ }^{*} p<0.05$, ${ }^{* *} p<0.01,{ }^{* * *} p<0.001$. c Immunofluorescence staining of p65 in CRYAB-overexpressing and control cells after TNF- $\alpha$ treatment (10 ng/ml) for indicated times. Expression of CRYAB inhibited p65 nuclear localization. All data are shown from three independent experiments

these effects. Noteworthily, CRYAB expression did not affect TNFa-induced IKK $\beta$ activation (phosphorylation) in colon cancer cells, indicating that CRYAB inhibits NF-KB activation by blocking action of IKK $\beta$.

We used an in vitro assay system to measure the activity of IKK $\beta$ from HT29 cells. Compared with unstimulated cells, TNF-astimulated HT29 cells had markedly increased IKK $\beta$-specific activity, and co-treatment of these cells with TAT-CRYAB significantly decreased the activity (Fig. 5b). When HCT116 cells were transfected with plasmids expressing HA-CRYAB and FlagIKK $\beta$, we found the co-immunoprecipitation of exogenously expressed HA-CRYAB with Flag-IKK $\beta$ and vice versa (Fig. $5 c$, d). Similarly, CRYAB also bound with IKKa when overexpressed in HCT116 cells (Fig. 5e, f). We further assessed the interactions of these molecules by co-transfecting plasmids expressing Flag-IKK $\beta$, HA-IKKa, and HA-CRYAB into HCT116 cells. As shown in Fig. 5g, h, the co-immunoprecipitation of Flag-IKK $\beta$ and HA-IKKa was markedly reduced in the presence of $H A-C R Y A B$ and vice versa. These results indicated that CRYAB likely inhibits IKK $\beta$ activity via binding to IKK $\beta$ and IKKa and interfering IKK complex formation.

Recombinant TAT-CRYAB protein has anti-inflammatory action and alleviates DSS-induced colitis in mice

To explore whether CRYAB could be used as an anti-inflammatory agent, the transduction domain of TAT, which is able to facilitate cell penetration of the recombinant heterogeneous proteins, ${ }^{25-27}$ was fused with CRYAB to generate a recombinant cell permeable CRYAB (Supplementary Fig. 3A) of near 29 KD (Supplementary Fig. 3B-C). HT29 and Caco-2 cells treated with recombined protein showed high levels of intracellular CRYAB (Fig. 6a). As presented in Fig. 6b, TNF- $a$ induced significantly less amounts of proinflammatory cytokines (e.g., TNF- $\alpha, \mathrm{IL}-6, \mathrm{IL}-1 \beta$, and IL-8) in TAT-CRYABtreated HT29 or Caco-2 cells. Notably, recombined TAT-CRYAB protein also inhibited the phosphorylation of IKBa and p65, and blocked IKBa degradation in these cells (Fig. $6 \mathrm{c}$ ).

The ability of TAT-CRYAB to inhibit proinflammatory signal and cytokine production encouraged us to determine whether the recombinant protein has an anti-inflammatory effect in an animal model of colitis. Mice fed with drinking water comprising of $3.5 \%$ DSS developed intestinal inflammation, manifested by a shortened colon, more severe colonic tissue damage and inflammatory cells infiltration, when compared with controls (there were five mice in the Water group and five mice in the DSS group, respectively; Supplementary Fig. 4A, B). Similar to the findings in UC and CD patients, CRYAB expression was significantly decreased in inflamed intestines from DSS-fed mice, as evidenced by immunoblotting (Supplementary Fig. 4C) and immunohistochemical analysis (Supplementary Fig. 4D).

As presented in Fig. 7a, a time-dependent increase of CRYAB in colon tissue from mice receiving intraperitoneal injection of TAT$C R Y A B$ recombined protein was observed, indicating that the recombined protein might penetrate into the colon cells (one mouse per group). Detailed injection process of the recombined protein and induction by DSS are presented in Fig. 7b (there were three mice in water + PBS group, three mice in water + TAT-CRYAB group, four mice in DSS + PBS group, and four mice in DSS + TAT-CRYAB group, respectively). In DSS-fed mice, the colitis mice treated with TAT-CRYAB recombined protein for 7 consecutive days appeared to be less severe compared with controls, as characterized by the shortened length of colons, a decrease of DAI score, and low levels of production of inflammatory cytokines (TNF- $\alpha, \mathrm{IL}-6, \mathrm{IL}-1 \beta$, and IL-8) (Fig. 7c-e). Compared with untreated mice, the colon tissues from mice receiving TAT-CRYAB recombined protein showed prominently intensified levels of CRYAB, reduced p65 and IKBa phosphorylation, and moderately decreased IKBa degradation (Fig. 7f). Similar results were also obtained from the immunohistochemical staining for CRYAB (Fig. 7g). Immunohistochemical staining for TAT revealed that intestinal tissues from mice treated with TATCRYAB were TAT positive, but negative in PBS-treated mice, suggesting that the administrated TAT-CRYAB indeed penetrated into the colon tissues (Fig. 7h). Moreover, hematoxylin-eosin staining revealed that colon tissues from TAT-CRYAB-treated mice had a decreased pathological score compared with controls (Fig. 7i). Collectively, these data demonstrate that recombinant TAT-CRYAB plays an anti-inflammatory role in protecting the intestines from inflammation.

Recombined TAT-CRYAB protein protects intestinal barrier integrity from DSS-induced damage

It has been shown that disruption of the intestinal barrier plays a critical role in the development of intestinal inflammation, which 
a

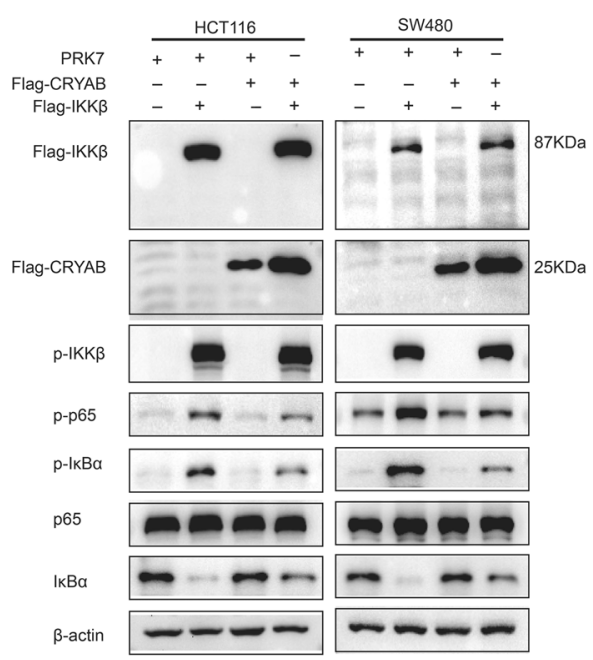

b

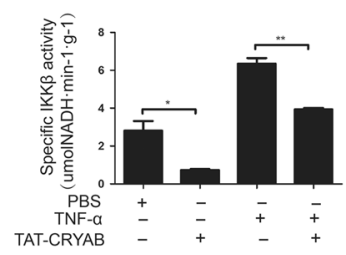

C
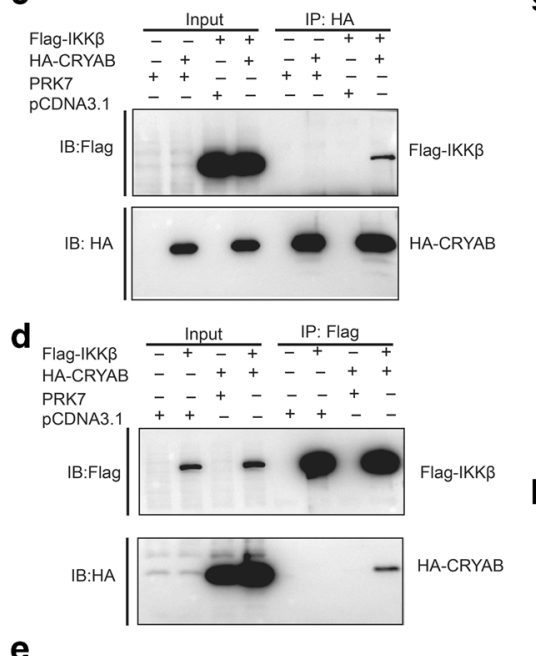

e

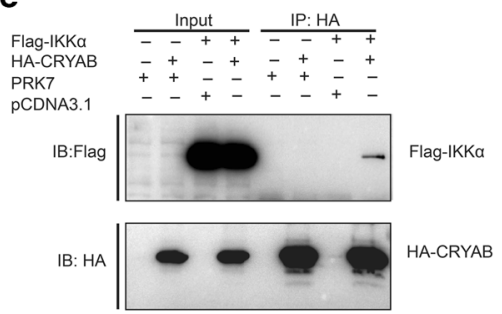

f

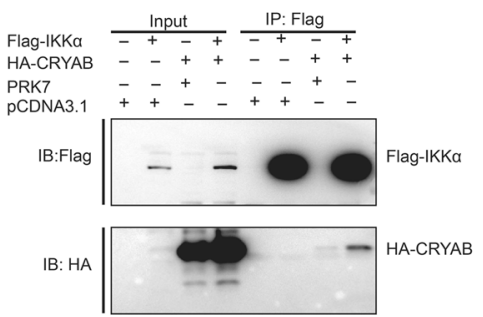

g

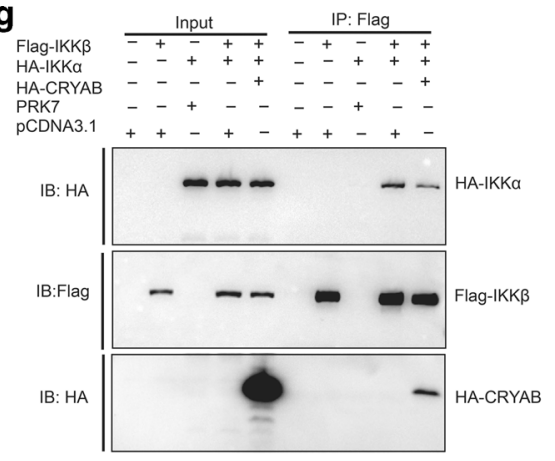

h
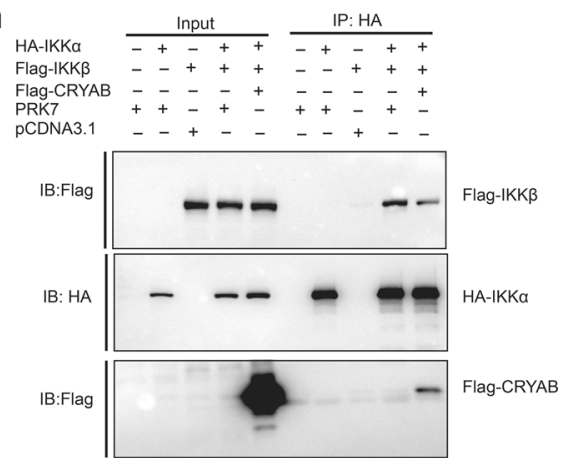

Fig. 5 CRYAB interacts with IKK $\beta$ and IKK $\alpha$ and inhibits IKK $\beta$ activity. a CRYAB inhibited IKK $\beta$-driven p65 phosphorylation, IKB $\alpha$ phosphorylation, and degradation. HCT116 and SW480 cells were transfected with control vector PRK7, Flag-IKK $\beta$, or Flag-CRYAB plasmids,

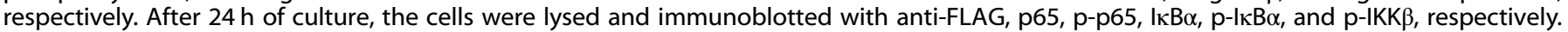
b CRYAB inhibits the IKK $\beta$ activity. HT29 cell was treated with TNF- $\alpha(10 \mu \mathrm{g} / \mathrm{ml})$ or control for $6 \mathrm{~h}$ in the absence or presence of pretreatment of TAT-CRYAB recombined protein $(5 \mu \mathrm{g} / \mathrm{ml})$ for $12 \mathrm{~h}$. Specific IKK $\beta$ activity was then measured using a peptide substrate and a small molecular IKK $\beta$ inhibitor; ${ }^{*} p<0.05,{ }^{* *} p<0.01$. c, d Co-immunoprecipitation of IKK $\beta$ and CRYAB. HCT116 cells were transfected to express HA-CRYAB and Flag-IKK $\beta$. Cell lysates were immunoprecipitated with anti-HA beads and blotted with anti-FLAG antibody or immunoprecipitated with anti-FLAG beads and blotted with anti-HA antibody. e, f Co-immunoprecipitation of IKK $\alpha$ and CRYAB. Cell lysates were immunoprecipitated with anti-HA beads and blotted with anti-FLAG antibody or immunoprecipitated with anti-FLAG beads and blotted with anti-HA antibody. g, h CRYAB inhibits the interaction between IKK $\alpha$ and IKK $\beta$. HCT116 cells were co-transfected with plasmids expressing Flag-IKK $\beta, \mathrm{HA}-\mathrm{IKK} \alpha$, and HA-CRYAB. Co-immunoprecipitation of expressed Flag-IKK $\beta$ and HA-IKK $\alpha$ was significantly inhibited compared with the cells without HA-CRYAB and vice versa. All data are shown from three independent experiments

is assessed by the amount of FITC-dextran in the blood after the mouse was fed the compound in drink water. As shown in Fig. 8a, administration of TAT-CRYAB significantly reduced the intestinal epithelial permeability, and ameliorated the goblet cell destruction in DSS-induced inflammatory intestines as evaluated by Alcian blue/periodic acid-Schiff staining (Fig. 8b). In the DSS-fed mice, there was also decreased ZO-1 expression, indicative of the destruction of epithelial tight junction, which was alleviated by administration of TAT-CRYAB (Fig. 8c). Colons from TAT-CRYABtreated mice also contained higher proteins levels of E-cadherin (Fig. 8d). Immunofluorescence staining of E-cadherin, CK20, and MUC2 further revealed that mice treated with TAT-CRYAB had less intestinal damage compared with those treated with PBS alone (Fig. 8e).

Noteworthily, administration of TAT-CRYAB also reduced TNBSinduced colitis in mice (there were three mice in absolute ethanol + PBS group, three mice in absolute ethanol + TAT-CRYAB group, four mice in TNBS + PBS group, and four mice in TNBS + TATCRYAB group, respectively; Supplementary Fig. 5) and protected the intestinal epithelial cells and integrity (Supplementary Fig. 6). Therefore, these data indicate that CRYAB functions as an effective therapeutic agent for intestinal inflammation.

\section{DISCUSSION}

In this study, we discovered that the small heat shock protein CRYAB modulated the sensitivity of intestinal cells to inflammatory stimuli. Increased $C R Y A B$ could inhibit the inflammatory responses, while lack of CRYAB markedly amplified the response. We also demonstrated that CRYAB itself was downregulated by a proinflammatory cytokine TNF-a (Fig. 3a), presumably due to initiating inflammatory response. Furthermore, CRYAB expression was significantly decreased in inflamed colon tissues of IBD patients, which correlated with increased inflammatory cytokines. 
a

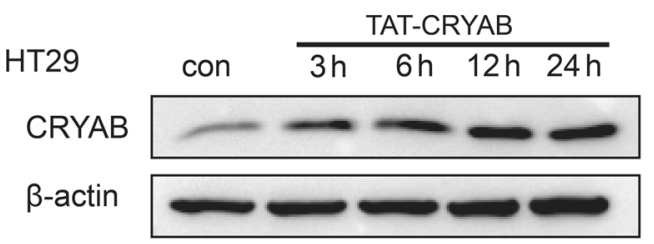

Caco-2

CRYAB

$\beta$-actin

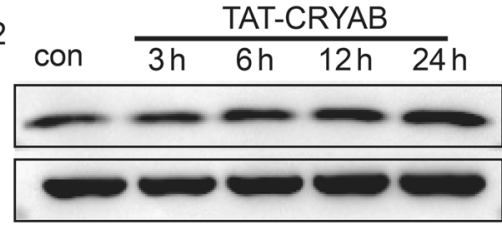

b

TNF- $\alpha$

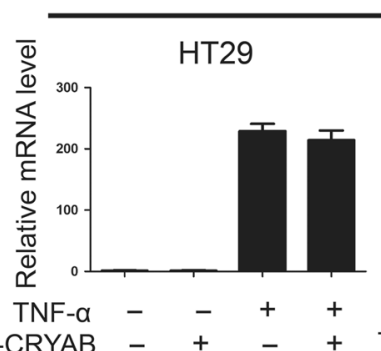

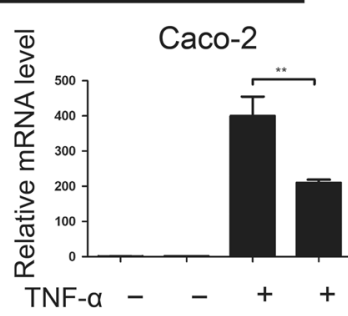

IL-6

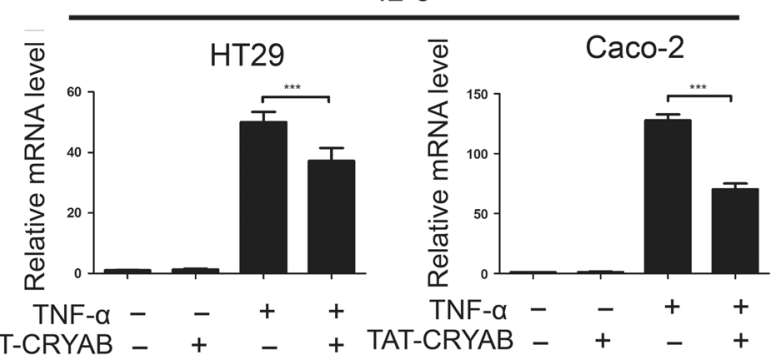

IL-1 $\beta$
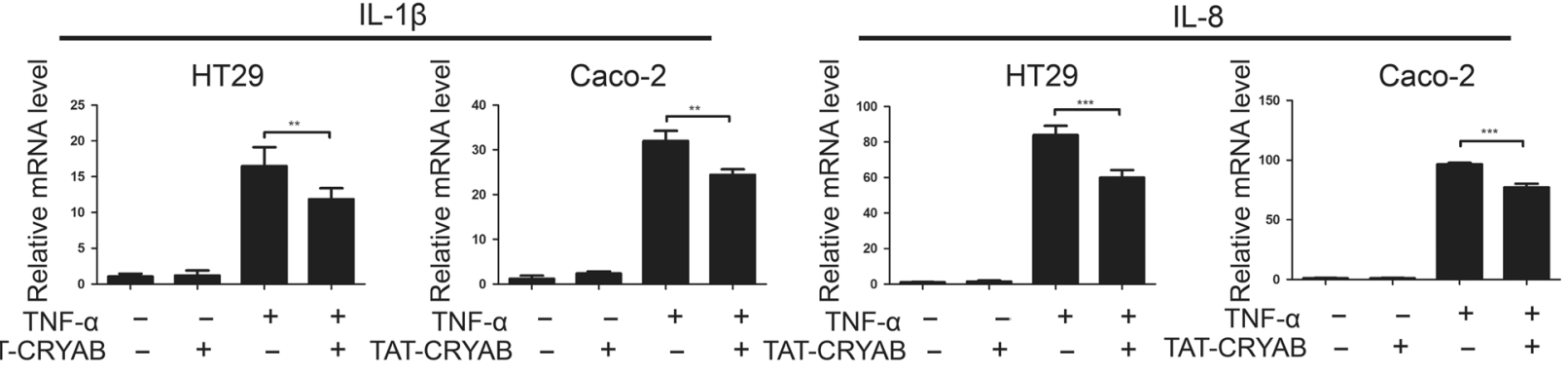

C

\section{HT29}

\section{Caco-2}

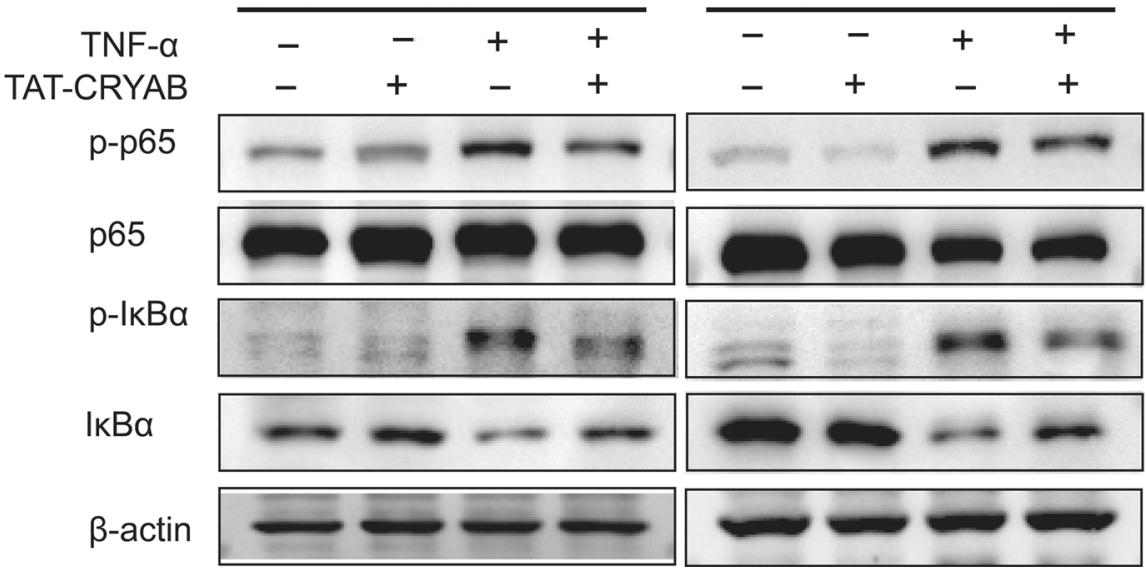

Fig. 6 Recombinant TAT-CRYAB protein inhibits inflammatory response of intestinal epithelial cells. a HT29 and Caco-2 cells were incubated with TAT-CRYAB $(500 \mathrm{ng} / \mathrm{ml})$ for indicated times. CRYAB expression was assessed by immunoblotting. b TNF- $\alpha$, IL-6, IL-1 $\beta$, and IL- 8 were inhibited by TAT-CRYAB treatment. HT29 and Caco-2 cells were treated with TNF- $\alpha(10 \mu \mathrm{g} / \mathrm{ml})$ for $6 \mathrm{~h}$ in the absence or presence of pretreatment of recombinant TAT-CRYAB $(5 \mu \mathrm{g} / \mathrm{ml})$ for $12 \mathrm{~h}$. The cytokine mRNAs were measured by qRT-PCR; ${ }^{*} p<0.05,{ }^{* *} p<0.01,{ }^{* * *} p<0.001$.

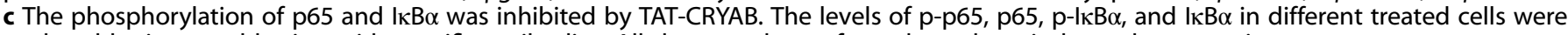
analyzed by immunoblotting with specific antibodies. All data are shown from three three independent experiments

Thus, our data indicate that CRYAB is crucial in the pathogenesis of IBD and may serve as a precision medicine biomarker to assess the disease and its response to anti-inflammatory effects.

CRYAB is a component of the small heat shock protein family, whose constituents are usually upregulated when cells are exposed to heat shock and other stresses, including accumulation of misfolded proteins in the cells. Noteworthily, heat shock proteins are often expressed at low-to-moderate levels in several cells, and function as chaperons to prevent unwanted protein aggregation. In this study, we observed that CRYAB inhibited the phosphorylation and degradation of $\mathrm{IKBa}_{\mathrm{K}}$, which is required for the activation of NF-kB, a critical transcription factor for the production of multiple proinflammatory cytokines. We further demonstrated that CRYAB did not affect the activation of IKK by inflammatory stimuli; however, it appeared to bind to IKK $\beta$ and inhibit its activity toward its substrate. We further found that CRYAB also interacts with IKKa and apparently blocks the formation of IKK complex to reduce the IKK $\beta$ activity. As the small heat shock proteins are characterized by a conserved a-crystallin-domain that participates in their interaction with partners, it is interesting to know in the future whether the a-crystallin-domain is involved in binding with IKK $\beta$ and IKKa and 
a

d

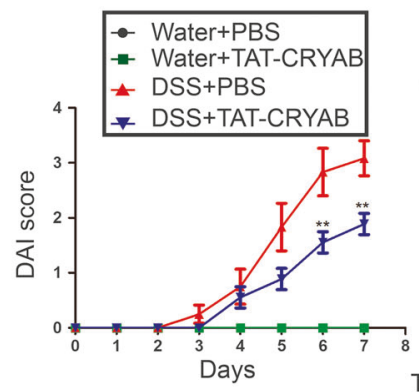

$\beta$-actin $-\infty-\infty$ b
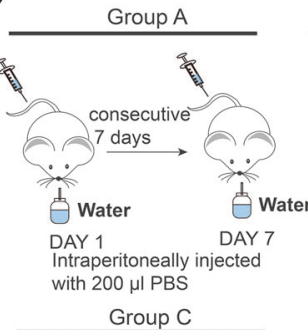

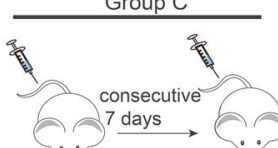

$\dot{2}$

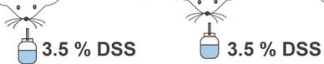

DAY 1 DAY 7

Intraperitoneally injected

with $200 \mu$ PBS
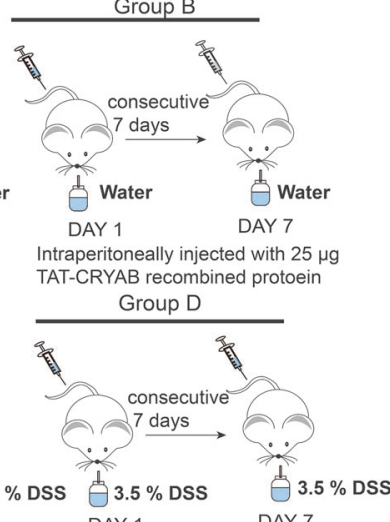

DAY 1

Intraperitoneally injected with $25 \mu \mathrm{g}$ TAT-CRYAB recombined protoein

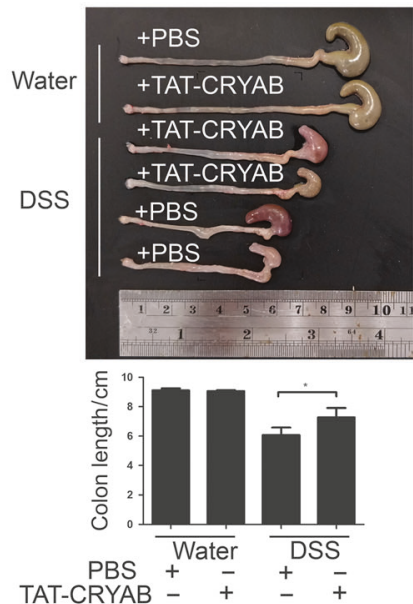

f

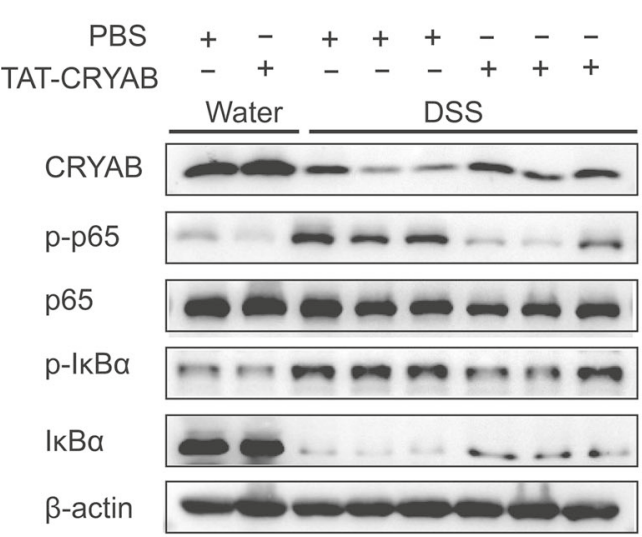

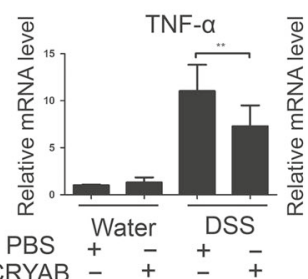

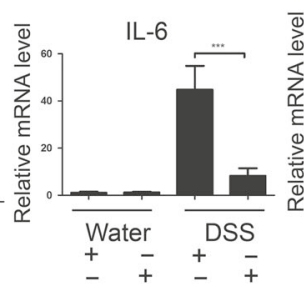

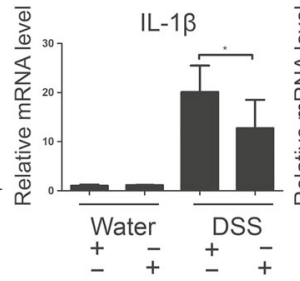

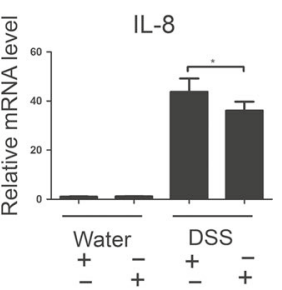

g

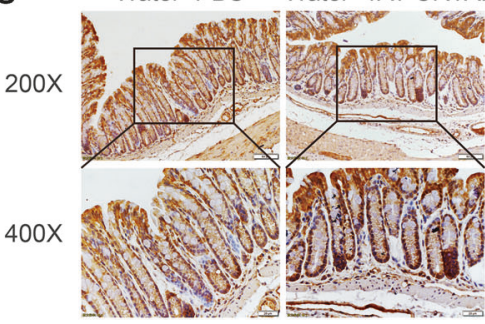

h

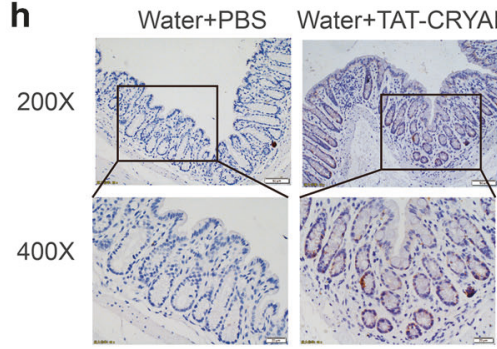

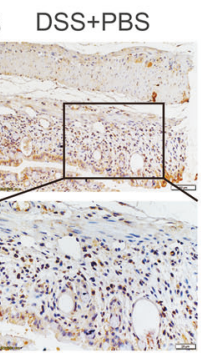

DSS+TAT-CRYAB

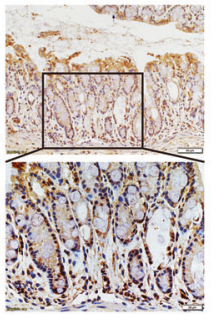

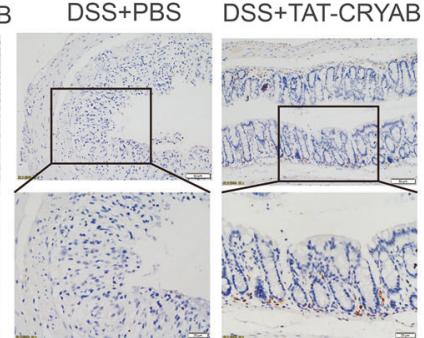

i
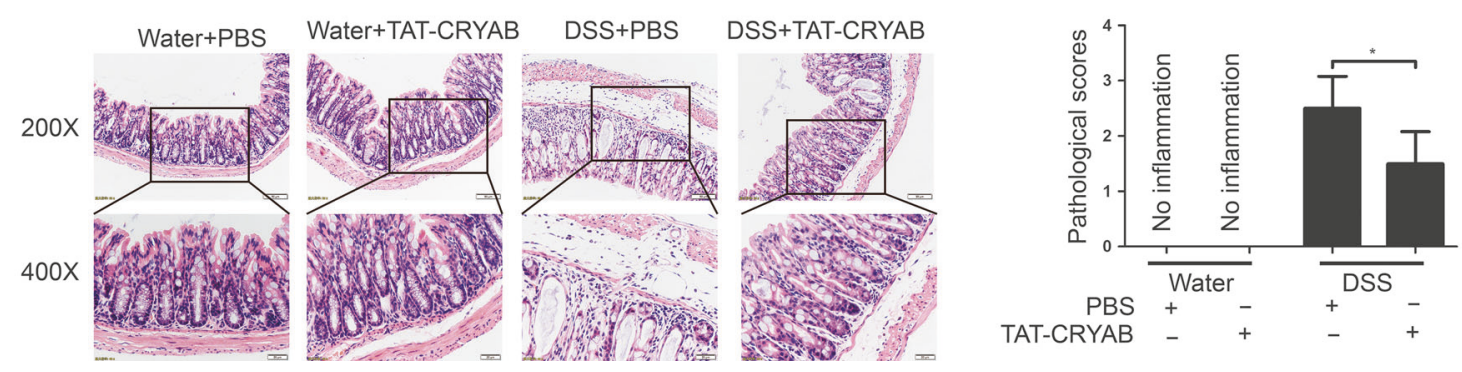

whether other small heat shock proteins have similar properties. Noteworthily, in contrast to the upregulation of heat shock proteins during cellular stress, CRYAB expression was downregulated in the cells exposed to inflammatory stimuli. Therefore,

better understanding the unique mechanisms may provide novel strategies for therapeutic intervention.

Consistent with our findings, previous research has demonstrated that $\mathrm{Cryab}^{-1-}$ mice are more likely to develop severe 
Fig. 7 Administration of TAT-CRYAB alleviates DSS-induced intestinal mucosal inflammation in mice. a Administration of TAT-CRYAB intraperitoneally increased CRYAB expression in the colon tissues. Each mouse was injected with $25 \mu \mathrm{g}$ of TAT-CRYAB intraperitoneally, and killed at indicated times to detect the levels of CRYAB in the colon (one mouse per group). $\mathbf{b}$ Schematic diagram of the TAT-CRYAB treatment in DSS-induced colitis (water + PBS group: three mice; water + TAT-CRYAB group: three mice; DSS + PBS group: four mice; DSS + TAT-CRYAB group: four mice). c TAT-CRYAB reduced colitis-induced shortening of colon length, and (d) DAI score; ${ }^{*} p<0.05,{ }^{* *} p<0.01$. e TNF- $\alpha$, IL-6, IL-1 $\beta$, and IL-8 in DSS-induced colitis were reduced by TAT-CRYAB administration. The cytokines in the colon were assessed by qRT-PCR; ${ }^{*} p<0.05$, ${ }^{* *} p<0.01,{ }^{* *} p<0.001$. f CRYAB, $p-p 65, p 65, p-1 \kappa B \alpha$, and $1 \kappa B \alpha$ in the colon were analyzed by immunoblotting with specific antibodies. g Representative immunohistochemical images of CRYAB expression in colons from different groups of mice (magnification: $\times 200$, upper panels; $\times 400$, lower panels). $\mathbf{h}$ Representative immunohistochemical images of TAT-CRYAB expression examined by the anti-TAT antibody in colons from different groups of mice (magnification: $\times 200$, upper panels; $\times 400$, lower panels). $\mathbf{i}$ Representative images of hematoxylin-eosin staining and pathological scores of colon tissues from DSS-exposed mice with or without TAT-CRYAB administration (magnification: $\times 200$, upper panels; $\times 400$, lower panels); ${ }^{*} p<0.05$. All data are shown from three independent experiments

a

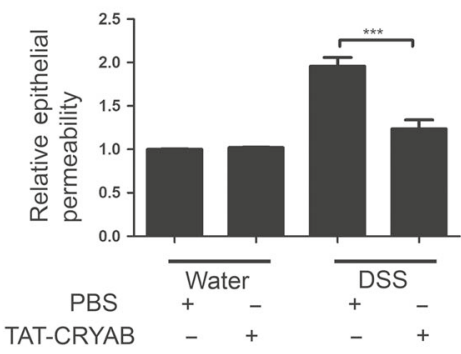

C

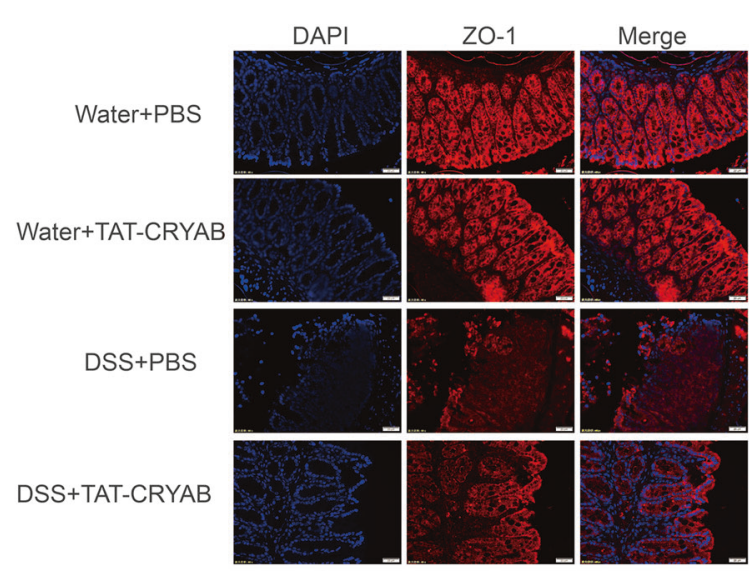

b

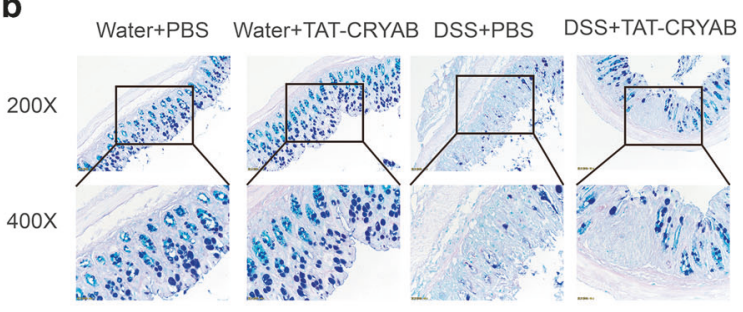

d
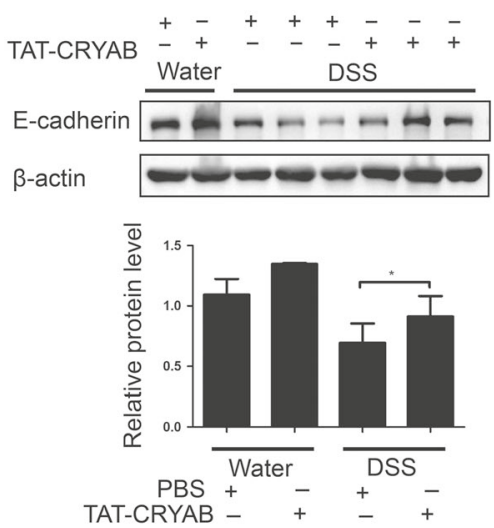

e

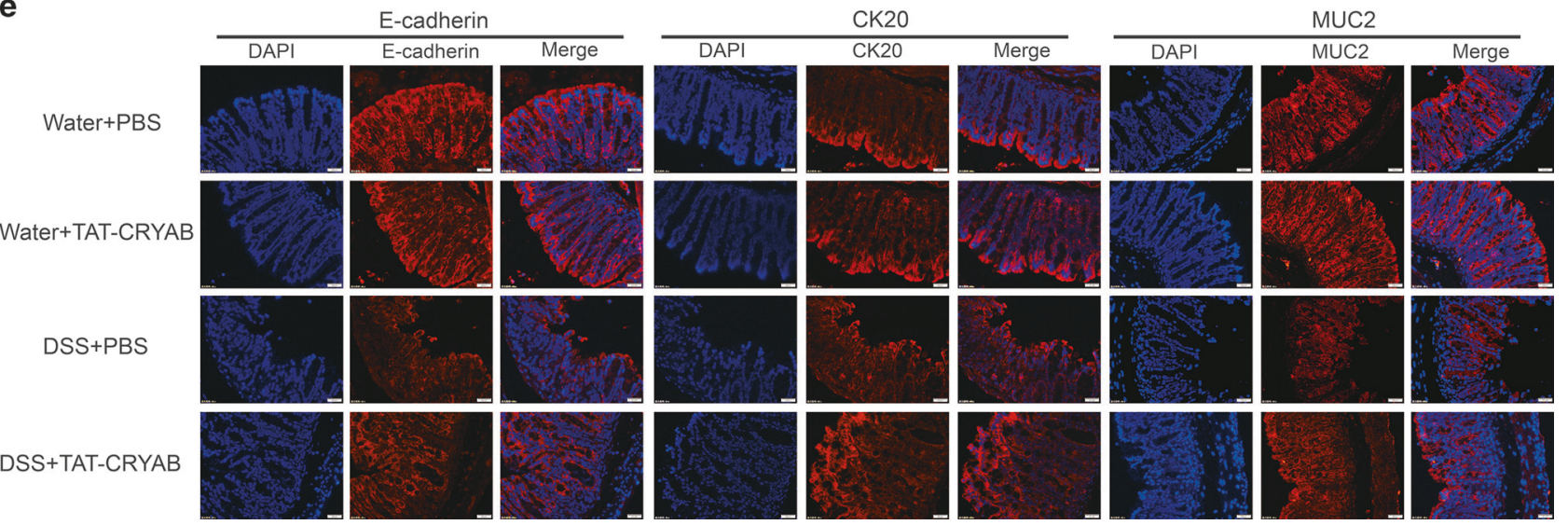

Fig. 8 TAT-CRYAB administration ameliorates DSS-induced intestinal damages. Water + PBS group: three mice; water + TAT-CRYAB group: three mice; DSS + PBS group: four mice; DSS + TAT-CRYAB group: four mice. a Epithelial permeability was measured on day 7 post-DSS treatment by oral administration of FITC-dextran. ${ }^{* * *} p<0.001$. b Representative images of Alcian blue/periodic acid-Schiff staining of colon tissues from mice of different groups (magnification: $\times 200$, upper panels; $\times 400$, lower panels). c Representative immunofluorescence images for ZO-1 in colonic tissues from different groups of mice (magnification: $\times 400$ ). d E-cadherin expression in colonic tissues from different groups was examined by immunoblotting; ${ }^{*} p<0.05$. e Representative immunofluorescence images for E-cadherin, CK20, and MUC2 in colonic tissues from different groups of mice (magnification: $\times 400$ ). All data are shown from three independent experiments 


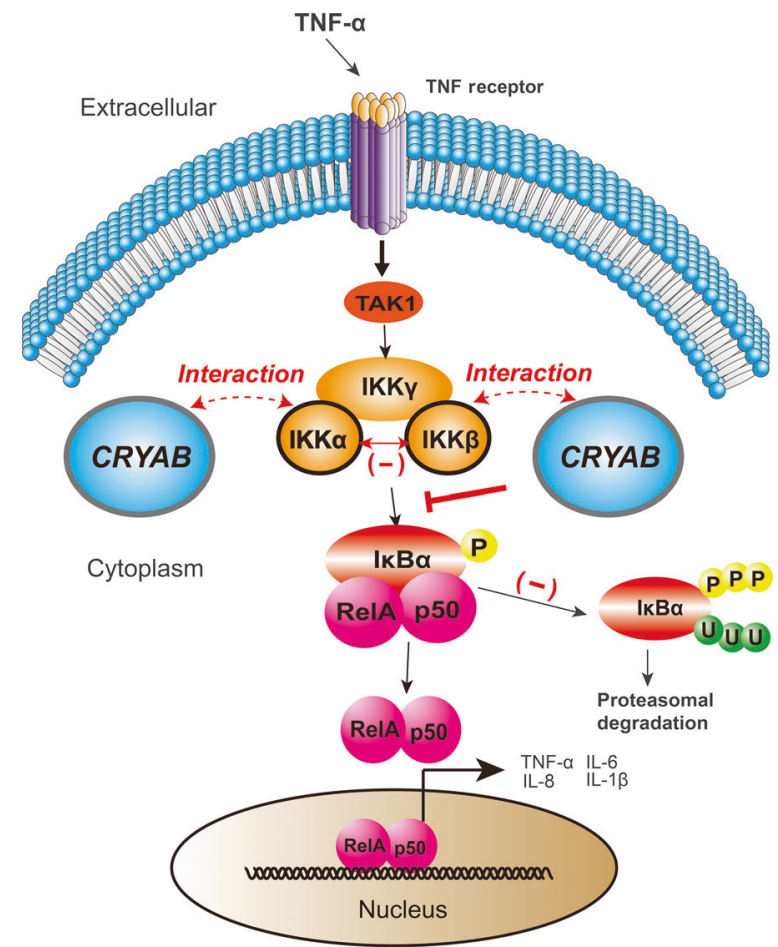

Fig. 9 Schematic diagram of CRYAB on the activation of the NF- $\mathrm{KB}$ pathway by TNF- $\alpha$. On TNF- $\alpha$ stimulation, the TNF- $\alpha$ receptor is activated, followed by the activation of TAK1, which is the crucial pivot for the important intersection of the NF-KB pathway activation. Thereafter, the activation of the IKK complex and subsequent degradation of $I_{\kappa} B \alpha$ via the phosphorylation catalytic subunit IKK $\beta$ are activated. According to the aforementioned results, we found that CRYAB does not affect the degradation of IKK complexes, but inhibits IKK $\beta$ activity via binding to IKK $\beta$ and IKK $\alpha$ and interfering IKK complex formation

experimental autoimmune encephalomyelitis and have increased immune activation and intense central nervous system inflammation. ${ }^{18}$ However, it was not determined whether there were any changes in spontaneous or induced intestinal inflammation in $\mathrm{Cryab}^{-1-}$ mice. Therefore, it is interesting to further investigate the role of $C R Y A B$ in regulating mucosal inflammatory and immune responses using DSS- and TNBS-induced colitis model in $\mathrm{Cryab}^{-1-}$ mice, especially conditional knockout models to assess the roles of CRYAB in myeloid cells and intestinal epithelial cells.

TNF- $a$ is crucial in the initiation and development of IBD. ${ }^{28-31}$ The successful therapy using various anti-TNF-a antibodies represents a major breakthrough in the treatment of IBD, and it also propelled the development of novel biologicals targeting additional cytokines and inflammation-related molecules. ${ }^{32}$ The infliximab ACCENT I clinical study, the CHARM study of adalimumab and the vedolizumab GEMINI II study have shown a complete 54 -week remission rate of $16.7 \%, 25.2 \%$, and $17.7 \%$, respectively. ${ }^{33-35}$ It is conceivable that involvement of multiple proinflammatory cytokines in IBD patients at different stages of disease is responsible for the lack of effects. Thus, the ability of CRYAB to inhibit IKK $\beta$ and the IKK complex formation and then block the production of multiple cytokines makes it a desirable therapeutic approach for anti-inflammatory treatment in IBD (Fig. 9).

It has been reported that the TAT-PDB domain is capable of mediating fused protein into cells and affects the cellular functions, including reprogramming and ROS generation. ${ }^{36-39}$ In this study, we generated a novel TAT-CRYAB fusion protein, and demonstrated for the first time that it not only had antiinflammatory effect in the cultured cells but also markedly alleviated DSS- and TNBS-induced colitis in mice, accompanied by improving the colon length, and downregulating DAI scores, histopathological scores, and inflammatory cytokine levels. In addition, the administration of TAT-CRYAB protected the integrity of the intestinal barrier and prevented ulcerative formation in murine colitis models. Thus, the recombinant protein is a promising novel therapeutic and needs to be further explored.

\section{CONCLUSIONS}

$C R Y A B$ is significantly decreased in inflamed mucosa of IBD patients and experimental colitis in mice. It appears to set the cellular sensitivity to inflammation. Enforced expression of CRYAB inhibits IKK $\beta$ through hindering the formation of active IKK complex and exerts anti-inflammatory effect, whereas CRYAB deficiency enhances inflammatory response. Thus, decreased CRYAB presumably contributes to the development of IBD. In addition, recombinant protein TAT-CRYAB possesses antiinflammatory property and alleviates mucosal inflammation, indicating that the fusion protein could be a novel therapeutic agent for the treatment of IBD.

\section{ACKNOWLEDGEMENTS}

This work was supported by the grant from the National Natural Science Foundation of China (81873547, 81570474, 81630017, and 91740117) and CAMS Initiative for Innovative Medicine (CAMS-I2M, 2016-12M-1-005).

\section{AUTHOR CONTRIBUTIONS}

Peng Du is a guarantor of the article. Peng Du, Yili Yang, and Zhanju Liu conceived and designed the study. Weimin $\mathrm{Xu}$, Yuegui Guo, and Zhenyu Huang performed all experiments. Weimin Xu wrote the final paper with input from Zhanju Liu, Yili Yang, and Peng Du. Haoxin Zhao, Mingxia Zhou, Yuji Huang, and Dongpeng Wen assisted with qRT-PCR experiments about inflammatory cytokines. Jinglue Song, Zhehui Zhu, and Mingming Sun collaborated to collect intestinal biopsies of IBD patients. ChenYing Liu, Yingwei Chen, Long Cui, and Xiaolei Wang analyzed the data. All authors reviewed the paper and approved the final version.

\section{ADDITIONAL INFORMATION}

The online version of this article (https://doi.org/10.1038/s41385-019-0198-5) contains supplementary material, which is available to authorized users.

Competing interests: All authors have read and approved the paper, and none of them have any conflict of interests to declare.

Ethical approval: All procedures performed in studies involving human participants were in accordance with the ethical standards of the institutional and/or national research committee and with the 1964 Helsinki declaration and its later amendments or comparable ethical standards. All animal experiments were in accordance with the U.K. Animals (Scientific Procedures) Act and its later amendments or comparable ethical standards.

Publisher's note: Springer Nature remains neutral with regard to jurisdictional claims in published maps and institutional affiliations.

\section{REFERENCES}

1. Kaplan, G. G. \& Ng S. C. Understanding and preventing the global increase of inflammatory bowel disease. Gastroenterology 152, 313-321.e312 (2017).

2. Molodecky, N. A. et al. Increasing incidence and prevalence of the inflammatory bowel diseases with time, based on systematic review. Gastroenterology 142, 46-54 e42 (2012). quiz e30.

3. Podolsky, D. Inflammatory bowel disease. N. Engl. J. Med. 347, 417-429 (2002).

4. Kaser, A., Zeissig, S. \& Blumberg, R. S. Inflammatory bowel disease. Annu. Rev. Immunol. 28, 573-621 (2010).

5. $\mathrm{Ng}, \mathrm{S}$. C. et al. Geographical variability and environmental risk factors in inflammatory bowel disease. Gut 62, 630-649 (2013).

6. Bernstein, C. \& Shanahan, F. Disorders of a modern lifestyle: reconciling the epidemiology of inflammatory bowel diseases. Gut 57, 1185-1191 (2008). 
7. Thia, K., Loftus, E., Sandborn, W. \& Yang, S. An update on the epidemiology of inflammatory bowel disease in Asia. Am. J. Gastroenterol. 103, 3167-3182 (2008).

8. Maloy, K. J. \& Powrie, F. Intestinal homeostasis and its breakdown in inflammatory bowel disease. Nature 474, 298-306 (2011).

9. Marchioni Beery, R. \& Kane, S. Current approaches to the management of newonset ulcerative colitis. Clin. Exp. Gastroenterol. 7, 111-132 (2014).

10. Gruvberger-Saal, S. K. \& Parsons, R. Is the small heat shock protein alphaBcrystallin an oncogene? J. Clin. Invest. 116, 30-32 (2006).

11. Lutsch, G. et al. Abundance and location of the small heat shock proteins HSP25 and alphaB-crystallin in rat and human heart. Circulation 96, 3466-3476 (1997).

12. Iwaki, T., Kume-Iwaki, A. \& Goldman, J. E. Cellular distribution of alpha B-crystallin in non-lenticular tissues. J. Histochem Cytochem. 38, 31-39 (1990).

13. Li, Q., Wang, Y., Lai, Y., Xu, P. \& Yang, Z. HspB5 correlates with poor prognosis in colorectal cancer and prompts epithelial-mesenchymal transition through ERK signaling. PLOS ONE 12, e0182588 (2017).

14. Shi, C., Yang, X., Bu, X., Hou, N. \& Chen, P. Alpha B-crystallin promotes the invasion and metastasis of colorectal cancer via epithelial-mesenchymal transition. Biochem Biophys. Res. Commun. 489, 369-374 (2017).

15. Chen, D. et al. Alpha B-crystallin promotes the invasion and metastasis of gastric cancer via NF-kappaB-induced epithelial-mesenchymal transition. J. Cell Mol. Med. 22, 3215-3222 (2018).

16. Huang, X. Y. et al. alphaB-crystallin complexes with 14-3-3zeta to induce epithelial-mesenchymal transition and resistance to sorafenib in hepatocellular carcinoma. Hepatology 57, 2235-2247 (2013).

17. Fung, G. et al. Phosphorylation and degradation of alphaB-crystallin during enterovirus infection facilitates viral replication and induces viral pathogenesis. Oncotarget 8, 74767-74780 (2017).

18. Ousman, S. S. et al. Protective and therapeutic role for alphaB-crystallin in autoimmune demyelination. Nature 448, 474-479 (2007).

19. Brownell, S., Becker, R. \& Steinman, L. The protective and therapeutic function of small heat shock proteins in neurological diseases. Front Immunol. 3, 74 (2012).

20. Whiston, E. A. et al. alphaB-crystallin protects retinal tissue during Staphylococcus aureus-induced endophthalmitis. Infect. Immun. 76, 1781-1790 (2008).

21. Arac, A. et al. Systemic augmentation of alphaB-crystallin provides therapeutic benefit twelve hours post-stroke onset via immune modulation. Proc. Natl Acad. Sci. USA 108, 13287-13292 (2011).

22. Zhang, Y. et al. Activation of dopamine D2 receptor suppresses neuroinflammation through alphaB-crystalline by inhibition of NF-kappaB nuclear translocation in experimental ICH mice model. Stroke 46, 2637-2646 (2015)

23. Ito, R. et al. Interferon-gamma is causatively involved in experimental inflammatory bowel disease in mice. Clin. Exp. Immunol. 146, 330-338 (2006).

24. Gupta, R. B. et al. Histologic inflammation is a risk factor for progression to colorectal neoplasia in ulcerative colitis: a cohort study. Gastroenterology 133, 1099-1105 (2007). quiz1340-1091.

25. Ziegler, A. \& Seelig, J. High affinity of the cell-penetrating peptide HIV-1 Tat-PTD for DNA. Biochemistry 46, 8138-8145 (2007).

26. Schwarze, S., Ho, A., Vocero-Akbani, A. \& Dowdy, S. In vivo protein transduction: delivery of a biologically active protein into the mouse. Science $\mathbf{2 8 5}, \mathbf{1 5 6 9 - 1 5 7 2}$ (1999).
27. Nagahara, H. et al. Transduction of full-length TAT fusion proteins into mammalian cells: TAT-p27Kip1 induces cell migration. Nat. Med. 4, 1449-1452 (1998).

28. Matsuda, R. et al. Quantitive cytokine mRNA expression profiles in the colonic mucosa of patients with steroid naïve ulcerative colitis during active and quiescent disease. Inflamm. Bowel Dis. 15, 328-334 (2009).

29. Komatsu, M. et al. Tumor necrosis factor-alpha in serum of patients with inflammatory bowel disease as measured by a highly sensitive immuno-PCR. Clin. Chem. 47, 1297-1301 (2001).

30. Rioux, J. et al. Genomewide search in Canadian families with inflammatory bowe disease reveals two novel susceptibility loci. Am. J. Hum. Genet. 66, 1863-1870 (2000).

31. Hampe, J. et al. Linkage of inflammatory bowel disease to human chromosome 6p. Am. J. Hum. Genet. 65, 1647-1655 (1999).

32. Melmed, G. \& Targan, S. Future biologic targets for IBD: potentials and pitfalls. Nat. Rev. Gastroenterol. Hepatol. 7, 110-117 (2010).

33. Feagan, B. et al. Efficacy of vedolizumab in fistulising crohn's disease: exploratory analyses of data from GEMINI 2. J. Crohns Colitis 12, 621-626 (2018).

34. Panaccione, R. et al. Adalimumab maintains remission of Crohn's disease after up to 4 years of treatment: data from CHARM and ADHERE. Aliment Pharm. Ther. 38, 1236-1247 (2013).

35. Cornillie, F. et al. Postinduction serum infliximab trough level and decrease of Creactive protein level are associated with durable sustained response to infliximab: a retrospective analysis of the ACCENT I trial. Gut 63, 1721-1727 (2014).

36. Zhang, $H$. et al. Reprogramming of somatic cells via TAT-mediated protein transduction of recombinant factors. Biomaterials 33, 5047-5055 (2012).

37. Pan, C., Jia, W., Lu, B. \& Bishop, C. E. Expression of TAT recombinant Oct4, Sox2, Lin28, and Nanog proteins from baculovirus-infected Sf9 insect cells. Gene 556, 245-248 (2015)

38. Lan, M. S., Chen, C., Saunee, N. A., Zhang, T. \& Breslin, M. B. Expression of biologically active TAT-fused recombinant islet transcription factors. Life Sci. 114 45-50 (2014).

39. Park, H. et al. Alpha B-crystallin prevents the arrhythmogenic effects of particulate matter isolated from ambient air by attenuating oxidative stress. Toxicol. Appl. Pharm. 266, 267-275 (2013).

Open Access This article is licensed under a Creative Commons Attribution 4.0 International License, which permits use, sharing, adaptation, distribution and reproduction in any medium or format, as long as you give appropriate credit to the original author(s) and the source, provide a link to the Creative Commons license, and indicate if changes were made. The images or other third party material in this article are included in the article's Creative Commons license, unless indicated otherwise in a credit line to the material. If material is not included in the article's Creative Commons license and your intended use is not permitted by statutory regulation or exceeds the permitted use, you will need to obtain permission directly from the copyright holder. To view a copy of this license, visit http://creativecommons. org/licenses/by/4.0/.

(c) The Author(s) 2019 